\title{
Efficient Traffic Load-Balancing via Incremental Expansion of Routing Choices
}

\author{
PING YIN, University of California, San Diego, USA \\ SEN YANG and JUN XU, Georgia Institute of Technology, USA \\ JIM DAI, Cornell University, USA \\ BILL LIN, University of California, San Diego, USA
}

\begin{abstract}
Routing policies play a major role in the performance of communication networks. Backpressure-based adaptive routing algorithms where traffic is load balanced along different routing paths on a per-packet basis have been studied extensively in the literature. Although backpressure-based algorithms have been shown to be networkwide throughput optimal, they typically have poor delay performance under light or moderate loads, because packets may be sent over unnecessarily long routes. Further, backpressure-based algorithms have required every node to compute differential backlogs for every per-destination queue with the corresponding per-destination queue at every adjacent node, which is expensive given the large number of possible pairwise differential backlogs between neighbor nodes. In this article, we propose new backpressure-based adaptive routing algorithms that only use shortest-path routes to destinations when they are sufficient to accommodate the given traffic load, but the proposed algorithms will incrementally expand routing choices as needed to accommodate increasing traffic loads. We show analytically by means of fluid analysis that the proposed algorithms retain networkwide throughput optimality, and we show empirically by means of simulations that our proposed algorithms provide substantial improvements in delay performance. Our evaluations further show that, in practice, our approach dramatically reduces the number of pairwise differential backlogs that have to be computed and the amount of corresponding backlog information that has to be exchanged, because routing choices are only incrementally expanded as needed.
\end{abstract}

CCS Concepts: • Networks $\rightarrow$ Routing protocols; Routers;

Additional Key Words and Phrases: Dynamic routing, multipath routing, traffic engineering, load balancing

ACM Reference format:

Ping Yin, Sen Yang, Jun Xu, Jim Dai, and Bill Lin. 2019. Efficient Traffic Load-Balancing via Incremental Expansion of Routing Choices. ACM Trans. Model. Perform. Eval. Comput. Syst. 4, 1, Article 1 (January 2019), 35 pages.

https://doi.org/10.1145/3243173

\footnotetext{
This work was supported in part by the National Science Foundation under grant CNS-1248117, grant CNS-1422286, and grant CNS-1423182.

Authors' addresses: P. Yin and B. Lin, University of California, San Diego, Department of Electrical and Computer Engineering, La Jolla, CA, 92093-0407; emails: \{piyin, billlin\}@eng.ucsd.edu; S. Yang and J. Xu, Georgia Institute of Technology, College of Computing, Atlanta, GA, 30332; emails: sen.yang@gatech.edu, jx@cc.gatech.edu; J. Dai, Cornell University, School of Operations Research and Information Engineering, Ithaca, NY, 14853; email: jd694@cornell.edu.

Permission to make digital or hard copies of all or part of this work for personal or classroom use is granted without fee provided that copies are not made or distributed for profit or commercial advantage and that copies bear this notice and the full citation on the first page. Copyrights for components of this work owned by others than ACM must be honored. Abstracting with credit is permitted. To copy otherwise, or republish, to post on servers or to redistribute to lists, requires prior specific permission and/or a fee. Request permissions from permissions@acm.org.

(C) 2019 Association for Computing Machinery.

2376-3639/2019/01-ART1 \$15.00

https://doi.org/10.1145/3243173
}

ACM Trans. Model. Perform. Eval. Comput. Syst., Vol. 4, No. 1, Article 1. Publication date: January 2019. 


\section{INTRODUCTION}

Traffic on the Internet continues to grow at a rapid pace. The choice of routing algorithm plays a vital role in the performance of communication networks. Compared with fixed routing [5, 15, 16], where each flow has a single and fixed route, adaptive routing is more appealing, because it offers better latency and throughput by routing packets along different routing paths, depending on network congestion. Among adaptive routing algorithms, backpressure-based algorithms [1$3,5,19-21,23,26,27]$ have been extensively studied in the literature, because they have been shown to be network-wide throughput optimal [23]. It was initially introduced in the context of wireless radio networks, but it can be easily adapted to wireline networks as well, for example for packet routing in backbone networks for the Internet. Despite throughput-optimality guarantees, backpressure-based algorithms have not been used in practice due to several shortcomings.

First, backpressure-based algorithms typically have poor delay performance under light or moderate loads, because packets may be sent over unnecessarily long routes, potentially traversing routing loops. The original backpressure algorithm allows the routing of a packet to any adjacent node as the next-hop, even if the routing decisions will cause a packet to take long detours. Second, backpressure algorithms typically maintain per-destination queues, and the routing and scheduling decisions are based on maintaining differential backlogs for every per-destination queue with the corresponding per-destination queue at every adjacent node. Although the implementation of per-destination queues has often been cited as a concern $[5,15,16,26]$, we note that significant advances have been made in memory architectures, since the original backpressure routing work for implementing huge packet buffers at line rates that support a very large number of logical queues $[14,22,24,25] .^{1}$

Despite these advances that address the practical implementation of per-destination queues, the need remains for backpressure-based algorithms to compute differential backlogs for every per-destination queue with the corresponding per-destination queue at every adjacent node. This computation is expensive given the large number of possible pairwise differential backlogs. Further, the computation requires many exchanges of backlog information between every pair of adjacent nodes for every pair of per-destination queues. The substantial amount of computations and associated information exchanges remains significant impediments for practical implementations.

To address the poor delay performance concern, the backpressure idea can be applied to a fixed routing problem, where packets are forced to use shortest paths [5]. However, limiting routing choices shrinks the network stability region and is thus not throughput optimal. As we shall later see in the evaluation section, limiting the routing choices to just shortest paths will cause the network to saturate much earlier than if all routing choices are permitted.

Several prior works $[10,11,18,19,26]$ have recognized the importance of favoring shorter paths instead of only considering shortest paths. However, all these approaches still require the computation of differential backlogs for all per-destination queues between every pair of neighboring nodes and the associated exchanges of backlog information. In comparison, our proposed algorithms only need a small number of the computations and backlog information exchanges as needed. Although the approach studied in Reference [26] further offers provably minimal-hop (MinHop) routing, their solution dramatically increases the number of queues that each node needs to maintain, since their approach requires per-hop queues for each destination. The dramatic increase in the number of queues makes the associated differential backlog computation problem even more difficult. Besides, the delay performance using their MinHop approach depends on the choices of

\footnotetext{
${ }^{1}$ Some of these memory architectures have been in commercial use in modern Internet routers to support per-class queuing or per-flow queuing, where the number of logical queues far exceeds the number of destinations needed for backpressure routing.
} 
parameters used. As we shall see later in the evaluation section, our approach offers comparable delay performance with the carefully tuned MinHop algorithm, while significantly reducing the information exchange frequency.

Other algorithms have been proposed to reduce the delay without using shortest paths. The authors of References [1,2] have proposed to replicate packets adaptively to build up gradients towards the destinations faster. Not only does this approach need a duplicate buffer for each perdestination queue in a router, but managing the original packets with their replicas can be difficult in practice. Besides, the number of backlog information exchanges and computations between every pair of per-destination queues in their algorithm is the same as in the original backpressure algorithm. The authors of Reference [6] proposed a class of enhanced BP algorithms by incorporating a general queue-dependent bias function, which basically uses the queue state information beyond one hop into the traditional backpressure algorithm. Although proven to be throughput optimal, their algorithms add the computation complexity to compute the bias function term in addition to what the original backpressure algorithm needs. Even their simplest BPnxt algorithm requires twice the amount of computations and backlog information exchanges as what the original backpressure algorithm requires. The other algorithm, BPmin, not only needs global queue state information but also requires $N$ times more computations than the original backpressure algorithm. Our approach achieves the same goal with a much simpler design that is dynamically expanding the routing choices as needed in response to the network congestion.

Further, there are prior works $[15,16,27]$ that aim to reduce the number of backlog information exchanges needed to compute the pairwise differential backlogs of per-destination queues. The authors of Reference [27] proposed a cluster-based back-pressure algorithm, where nodes are grouped into clusters and each node needs only to maintain per-destination queues for destinations within its cluster. However, this approach, although retaining the same optimal throughput as the original backpressure algorithm, still has large delay at low or moderate traffic loads. The approaches in References $[15,16]$ eliminate the per-flow information by using per-hop queues and per-link queues, however, it can only be applied to fixed routing scenarios, while our approach aims at adaptive routing, where the path that a packet traverses in the network is not pre-defined.

Finally, the authors of References [3,5] introduced the idea of shadow queues for making adaptive routing decisions. Their idea is to create a shadow network in which a backpressure algorithm is used to make routing decisions. Although their approach does not require per-destination queuing of packets, their solution still incurs the same calculation complexity as the original backpressure algorithm for the shadow queues in that the same computation of differential backlogs for all destinations between every pair of neighboring nodes and the same associated exchanges of backlog information are still required. Further, although their approach stores the actual packets in per-neighbor queues instead of per-destination queues, the amount of packet buffer storage that each node needs remains the same. ${ }^{2}$ We believe that our solution is complementary to References $[3,5]$ in that the algorithms described in this article can be used as the shadow algorithm in their framework.

Although these algorithms partly addressed the aforementioned disadvantages of the original backpressure algorithm, to the best of our knowledge, no work has addressed all the aforementioned issues in an adaptive routing scenario. Our approach substantially improves the delay performance, significantly reduces the number of pairwise differential backlog information exchanges and computations, but still retains the same optimal throughput as the original backpressure algorithm.

\footnotetext{
${ }^{2}$ The state-of-the-art DRAM-based packet buffers [14, 22, 24, 25] can store a huge number of packets, tens of gigabytes, and support a very large number of logical queues.
} 


\subsection{Our Approach}

In this article, we propose two related classes of modified backpressure-based routing algorithms that address the aforementioned concerns. We first propose route-expanding backpressure-based routing algorithms that are based on the idea that routing choices should be limited to next-hops that are along shortest path routes by default. This approach significantly reduces the amount of differential backlog calculations and associated information exchanges as each node only has to consider a subset of next hops for each destination. In addition, this approach addresses delay performance concerns by only routing packets along shortest path routes when the traffic load is light or moderate.

In particular, we propose to detect congestion by monitoring per-destination queue lengths or the waiting times of packets in the per-destination queues. When the length of a per-destination queue or the waiting time of a packet at the head of a per-destination queue exceeds some threshold, the routing choices for the corresponding per-destination queue get expanded to include next hops that are not along shortest path routes. This expansion of routing choices is on a perdestination queue basis. Although a packet may be forwarded to a next hop that is not along a shortest path route to the destination, the packet may still be forwarded along a shortest path route from this next hop to the destination if the corresponding per-destination queue at this next hop is not yet congested. This way, routing choices are incrementally expanded at different nodes in the network as needed with increasingly longer paths considered. In effect, a packet can take a detour whenever it encounters congestion along the way to the destination. When a node expands its routing choices for packets for a particular destination, it notifies other adjacent nodes to begin providing backlog information, and it expands its differential backlog calculations with those adjacent nodes as well.

Alternatively, we propose semi-oblivious backpressure-based routing algorithms that simply route packets obliviously by default, which only consider shortest-path next-hops. In this default oblivious-routing mode, backlog information exchanges and differential backlog calculations are largely avoided, and only shortest-path next-hops are considered, which also addresses delay performance concerns by only routing packets along shortest path routes when the traffic load is light or moderate. The proposed semi-oblivious approach detects congestion by monitoring output port queue lengths. When the length of an output queue at a router exceeds some threshold, the entire router switches to backpressure-routing mode where packets are adaptively routed to any adjacent node as possible next-hops using the backpressure routing algorithm. Although when a router is in backpressure-routing mode, a packet may be forwarded to a next hop that is not along a shortest path route to the destination, the packet may still be forwarded along a shortest path route from this next hop forward if the subsequent routers are still in oblivious-routing mode. This way, the proposed semi-oblivious approach also incrementally expands routing choices as needed with increasingly longer paths considered.

\subsection{Contributions and Outline of the Paper}

The main contributions of this article are as follows:

- We propose two route-expanding BP (backpressure) algorithms, called L-BP and A-BP, that are based on the incremental expansion of backpressure routing choices in response to congestion at a node on a per-destination queue basis. L-BP detects congestion by monitoring per-destination queue lengths, whereas A-BP detects congestion by monitoring the waiting times of packets at the heads of per-destination queues. Networks running these two algorithms are purely backpressure-based, but some per-destination queues are in shortest-path mode while others are allowed to be forwarded to any neighbor node. 
- We also propose two semi-oblivious BP (backpressure) algorithms, called O-BP and E-BP, that combine OSPF and ECMP oblivious routing, respectively, with backpressure routing. Networks running these algorithms first use OSPF or ECMP oblivious routing when the traffic load is light or moderate, but these algorithms incrementally switch nodes on a pernode basis to backpressure routing in response to congestion detected by monitoring output port queue lengths.

- We prove theoretically that all these algorithms are network-wide throughput optimal (i.e., the proposed algorithms can explore the same network stability region as the original backpressure algorithm). In particular, we use a fluid model for our proofs, which models well the system dynamics of our modified algorithms.

- We extensively evaluate our proposed algorithms on the adaptive Internet routing problem. We show our evaluations on the Abilene network [13], a public PoP-level academic network in the US, using actual traffic profiles measured on the network. Our simulation results show that our proposed algorithms indeed provide substantial improvements in delay performance. Our simulation results further show that in practice, our approach dramatically reduces the number of pairwise differential backlogs that have to be computed and the amount of corresponding backlog information that has to be exchanged, because routing choices are only incrementally expanded as needed. That is, only a subset of per-destination queues in a subset of nodes (in the case of route-expanding BP algorithms) or a subset of nodes (in the case of the semi-oblivious BP algorithm) need to consider expanded routing choices even for traffic loads that approach the edge of the network stability region.

- To the best of our knowledge, our approach is the first work that substantially improves the delay performance and also significantly reduces the number of pairwise differential backlog information exchanges and computations, while keeping the same optimal throughput benefit as the original backpressure algorithm in the adaptive routing scenarios.

The rest of the article is organized as follows: In Section 2, we present the basic network model and summarize the original backpressure algorithm. Then we present our route-expanding BP algorithms and semi-oblivious BP algorithms in Sections 3 and 4, respectively. In Section 5, we use fluid analysis to prove that all these algorithms are throughput optimal. In Section 6, we describe our experimental setup and simulation results. We conclude our article in Section 7.

\section{BACKGROUND}

\subsection{The Network Model}

We consider a multi-hop network represented by a directed graph $\mathcal{G}=(\mathcal{N}, \mathcal{L})$, where $\mathcal{N}$ is the set of nodes and $\mathcal{L}$ is the set of directed links. All packets that enter the network are associated with a particular commodity that corresponds to the packet destination. A packet that is destined for node $c$ is regarded as a commodity $c$ packet, $c=1, \ldots, N$. We use $\mathcal{L}_{c}$ to denote the routing restrictions for commodity $c$, which is the set of all links $(a, b)$ that a commodity $c$ packet is allowed to use. ${ }^{3}$ Obviously, if there is no routing restriction for commodity $c$ packets, then $\mathcal{L}_{c}=\mathcal{L}$. The link capacity $\mu_{a b}(t)$ for link $(a, b)$ is defined to be the maximum number of packets that can be transmitted over link $(a, b)$ in one timeslot. ${ }^{4}$ In general, multiple commodities might be transmitted over this link during a single timeslot, but the total rate cannot exceed the link capacity $\mu_{a b}(t)$.

\footnotetext{
${ }^{3}$ As we shall see in Sections 3 and 4, our routing algorithms restrict routing choices to only shortest-path output links when the traffic is not heavy to minimize end-to-end delay. These routing restrictions are denoted by $\mathcal{L}_{c}$.

${ }^{4}$ Although we define $\mu_{a b}(t)$ here in terms of number of packets, our algorithms and results are applicable to any unit of data as appropriate for the intended application (e.g., the unit of data can just be bits or a rate).
} 
Each node $i$ maintains a set of internal queues for storing network layer packets according to their commodity. Therefore, the internal queues are also known as per-destination queues. In the remainder of this article, we will use internal queues and per-destination queues interchangeably.

Let $A_{n}^{(c)}(t)$ represent the cumulative amount of new commodity $c$ packets that exogenously arrives to source node $n$ by timeslot $t$ (since time 0 ). Assume these arrival processes are admissible. Let $D_{a b}^{(c)}(t)$ be the cumulative amount of commodity $c$ packets sent from node $a$ to node $b$ via link $(a, b)$ by timeslot $t$ (since time 0 ), $a, b, c=1, \ldots, N$.

Let $Q_{n}^{(c)}$ denote the internal queue in node $n$ that stores packets destined for node $c$. With a slight abuse of notation, let $Q_{n}^{(c)}(t)$ also represent the current backlog of commodity c packets stored in an internal queue at node $n$. The queue backlog $Q_{n}^{(c)}(t)$ contains packets that arrived exogenously by $A_{n}^{(c)}(t)$ as well as packets that arrived endogenously from other nodes by $D_{a n}^{(c)}(t), a=1, \ldots, N$. We define $Q_{c}^{(c)}(t)=0$ and $D_{c n}^{(c)}(t)=0$ for all $t, c=1, \ldots, N$ and $n=1, \ldots, N$, so that any packet that has been delivered to its destination is assumed to exit the network right away. The queue backlogs then satisfy the following equation for all $n=1, \ldots, N$ and $c=1, \ldots, N$ such that $n \neq c$,

$$
Q_{n}^{(c)}(t)=Q_{n}^{(c)}(0)-\sum_{b=1}^{N} D_{n b}^{(c)}(t)+\sum_{a=1}^{N} D_{a n}^{(c)}(t)+A_{n}^{(c)}(t) .
$$

\subsection{The Backpressure Algorithm}

The original backpressure algorithm was first introduced in Reference [23] in the context of wireless radio networks. It has been shown to achieve optimal throughput [23] and can serve as a solution to certain multi-commodity flow problems [4].

For each link $(a, b)$, the algorithm defines the optimal commodity $c_{a b}^{*}(t)$ as the commodity that maximizes the differential backlog (ties broken arbitrarily):

$$
c_{a b}^{*}(t) \triangleq \arg \max _{\left\{c \mid(a, b) \in \mathcal{L}_{c}\right\}}\left[Q_{a}^{(c)}(t)-Q_{b}^{(c)}(t)\right]
$$

and defines $W_{a b}^{*}(t)$ as the corresponding optimal weight:

$$
W_{a b}^{*}(t) \triangleq \max \left[Q_{a}^{\left(c_{a b}^{*}(t)\right)}(t)-Q_{b}^{\left(c_{a b}^{*}(t)\right)}(t), 0\right] .
$$

If $W_{a b}^{*}(t)>0$, then the internal commodity $c_{a b}^{*}(t)$ queue is scheduled to be served, and the packets will be transmitted over link $(a, b)$ during timeslot $t$. Otherwise, no packets will be transmitted over link $(a, b)$ during timeslot $t$.

It is common in wireless networks that only a subset of all links, referred to as a schedule, can transmit packets simultaneously due to interference. Let $\mathcal{S}$ be the set of all possible schedules. The original backpressure algorithm finds the optimal schedule, $S^{*}(t) \in \mathcal{S}$ as an optimization problem as follows:

$$
S^{*}(t)=\arg \max _{S \in \mathcal{S}} \sum_{(a, b) \in S} W_{a b}^{*}(t) \mu_{a b}(t) .
$$

At each timeslot $t$, for each link $(a, b) \in S^{*}(t), \mu_{a b}(t)$ packets are removed from $Q_{a}^{\left(c_{a b}^{*}\right)}$ and transmitted to $Q_{b}^{\left(c_{a b}^{*}\right)}$. If $Q_{a}^{\left(c_{a b}^{*}\right)}$ does not have $\mu_{a b}(t)$ packets, then all packets will leave $Q_{a}^{\left(c_{a b}^{*}\right)}$. For wireline networks, $\mu_{a b}(t)$ is a typically constant (e.g., one packet per timeslot), and $\mathcal{S}$ is always the set of all links, since all links can be activated without interfering with each other.

The intuition behind the backpressure algorithm is that packets may not be transmitted if the differential backlog is non-positive, which indicates a congestion at the downstream node. The original backpressure algorithm considers $\mathcal{L}_{c}=\mathcal{L}$ for any commodity $c$ packet. That is, any packet in node $a$, no matter what commodity it belongs to, can transmit to any neighbor node of node $a$, 


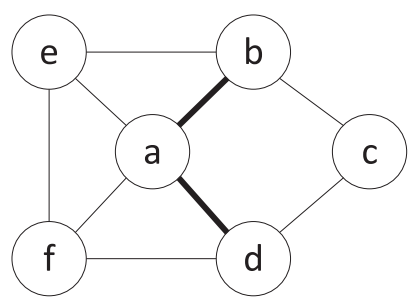

(c)

(a) Queue $Q_{a}$ is initially in Phase I.

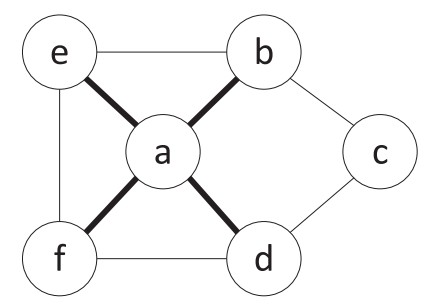

(c)

(b) Queue $Q_{a}$ switches over to Phase II.

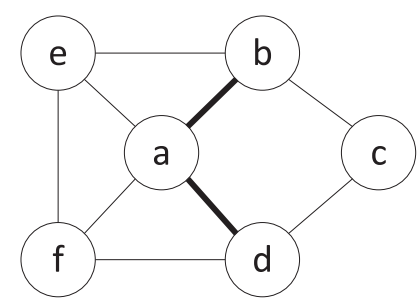

(c)

(c) Queue $Q_{a}$ returns back to Phase I.

Fig. 1. A simple network showing how $\mathcal{L}_{c}$ (thick edges) changes.

as long as the optimal weight computed by Equation (3) is positive. This feature essentially exploits all feasible paths in the network for any commodity packet and, as a result, stabilizes the network under heavy traffic loads. However, this feature also incurs large end-to-end packet delays when the network is only lightly or moderately loaded, because packets unnecessarily explore and traverse long paths.

In the following sections, we will use the original backpressure algorithm as a baseline algorithm to compare with our proposed algorithms.

\section{ROUTE-EXPANDING BP ALGORITHMS}

As mentioned in Section 2.2, the original backpressure algorithm assumes that $\mathcal{L}_{c}$ contains all of the links of the network, $\mathcal{L}$. This unconstrained routing may introduce large delays when the traffic load is light, as a packet can unnecessarily explore long paths.

One way to reduce the end-to-end delay is to restrict $\mathcal{L}_{c}$ to only shortest paths. We call this Shortest-Path Backpressure algorithm (SPBP). Take the network shown in Figure 1(a) as an example, node $c$ has two neighbors, $b$ and $d$. Neighbor $d$ is on the shortest path for commodity $f$ packets, while neighbor $b$ is not. In the case of SPBP, commodity $f$ packets in node $c$ can only transmit to node $d$ on condition that $f$ is the optimal commodity for link $(c, d)$ and its weight, computed by Equation (3), is positive. In comparison, the original backpressure algorithm allows commodity $f$ packets to transmit to both node $b$ and $d$, as long as $f$ is the optimal commodity for each link and the weight is positive.

While SPBP can reduce the delay, it also shrinks the network stability region, as it limits the routing choices compared with the original backpressure algorithm. However, our route-expanding backpressure algorithms can retain the same stability region as the original backpressure algorithm and reduce delay for light or moderate traffic loads by incremental expansion of routing choices. It starts with the shortest-path routing choices as described above for SPBP. To overcome its shortcomings, a dynamic change of routing choices is introduced.

In particular, each internal queue in a node $n$ has two phases of routing. A queue in Phase I can switch over to Phase II when a transition criterion is satisfied. A Phase II queue can also return Phase I when the transition criterion is no longer met. Similarly to the SPBP, packets in a Phase I queue can only go to a subset of the neighbor nodes, which are on the shortest paths from current node to the destination. In Phase II, similarly to the original backpressure algorithm, packets in that queue can be transmitted to any neighbor of the current node. The rest of the backpressure scheduling rules are the same, following Equations (2), (3), and (4).

The routing choices $\mathcal{L}_{c}$ are changing dynamically. In the beginning, all internal per-destination queues are in Phase I. This is equivalent to restricting $\mathcal{L}_{c}$ to allow only links on the shortest paths. When a transition criterion is satisfied, queue $Q_{n}^{(c)}$ switches over to Phase II, and we add all $(n, k)$ 
to set $\mathcal{L}_{c}$ for any neighbor $k$ of node $n$. When the transition criterion is no longer satisfied, $Q_{n}^{(c)}$ returns back to Phase I, and we remove those added links from $\mathcal{L}_{c}$. When all queues in the network are in Phase II, the $\mathcal{L}_{c}$ becomes $\mathcal{L}$, and this is equivalent to the original backpressure algorithm.

Consider the network shown in Figure 1 as an example and consider queue $Q_{a}^{(c)}$. In the beginning, $(a, b),(a, d) \in \mathcal{L}_{c}$, because node $b$ and node $d$ are on shortest paths to node $c$. When $Q_{a}^{(c)}$ switches over to Phase II, $(a, e),(a, f)$ are added to $\mathcal{L}_{c}$. When $Q_{a}^{(c)}$ returns back to Phase I, $(a, e)$, $(a, f)$ are then removed from $\mathcal{L}_{c}$.

We propose two transition criteria, a length-based criterion and an age-based criterion, which we refer to the corresponding algorithms as L-BP and A-BP, respectively:

- L-BP: Let $L_{\max }$ be the maximum backlog that a queue $Q_{n}^{(c)}$ can stay in Phase I. Whenever $Q_{n}^{(c)}(t)>L_{\max }$, the queue $Q_{n}^{(c)}$ switches over to Phase II. Whenever $Q_{n}^{(c)}(t) \leq(1-\epsilon) L_{\max }$, it returns back to Phase I. $\epsilon$ can be chosen to provide a safe margin between the transition thresholds to prevent a per-destination queue from transitioning back and forth frequently between the two phases ( $\epsilon$ can be any number in $[0,1)$; e.g., in the evaluation section, we use $\epsilon=0)$.

- A-BP: Consider the head packet of queue $Q_{n}^{(c)}$. Let $E_{n}^{(c)}(t)$ represent the age of the head packet, which is the period from the timeslot that the head packet enters the queue until current timeslot $t$. Let $A_{\max }$ be the maximum age of the head packet for its queue to stay in Phase I. Whenever $E_{n}^{(c)}(t)>A_{\max }$, the queue switches over to Phase II. Whenever $E_{n}^{(c)}(t) \leq$ $(1-\epsilon) A_{\text {max }}$, it returns back to Phase I. Like L-BP, $\epsilon$ can be chosen to provide a safe margin between the transition thresholds to prevent a per-destination queue from transitioning back and forth frequently between the two phases. In the evaluation section, we use $\epsilon=0$.

\section{SEMI-OBLIVIOUS BP ALGORITHMS}

In this section, we introduce two semi-oblivious backpressure-based routing algorithms called O$\mathrm{BP}$ and E-BP. Like L-BP and A-BP, the nodes in the network have two phases of routing.

In Phase I, nodes obliviously forward packets using OSPF (O-BP) or ECMP (E-BP). In OSPF [17], a shortest-path next-hop is identified for each destination, and incoming packets are obliviously forwarded to this shortest-path next-hop. In ECMP [12], all equal-cost shortest-path next-hops are identified for each destination, and incoming packets are obliviously routed to one of these shortest-path next-hops with equal probability.

When congestion is detected by monitoring output port queue lengths, a node transitions to Phase II in which the node will start forwarding packets based on the original backpressure routing policy, as described in Section 2, in which packets can be forwarded to any next-hop, not just shortest-path next-hops. A node remains in Phase II until the alleviation of congestion is detected, which can also be detected by monitoring output port queue lengths. A key benefit of using O$\mathrm{BP}$ or E-BP over L-BP/A-BP is that the number of backlog information exchanges and differential backlog calculations is largely avoided (packets are simply routed obliviously to a shortest-path next-hop) when O-BP and E-BP are in Phase I. In contrast, L-BP and A-BP require backlog information exchanges and differential backlog calculations for all per-destination queues between the current node and those neighbors that are on a shortest path of a packet. These information exchanges and calculations can be substantial.

However, a major challenge in combining OSPF or ECMP with backpressure routing is how packets are stored in a router. For OSPF, packets are queued at the output port that corresponds to a shortest path and served on a first-come-first-serve basis. For ECMP, packets are queued at multiple output ports that correspond to multiple shortest-path next-hops (if there is more than 
one shortest path), and packets are served at these output ports on a first-come-first-serve basis. As a result, one output port queue will likely have a mixture packets with many different destinations. However, in backpressure routing, packets are stored in per-destination queues according to their destination rather than their next hop (all packets in the same per-destination queue must have the same destination).

To overcome this incompatibility, we extend a router to implement both output port queues used in OSPF/ECMP and per-destination queues used in the backpressure policy. However, these output port queues and per-destination queues are simply implemented as doubly linked lists with pointers to packets rather than storing the actual packet themselves. This way, each packet is still only physically stored once. That is, $Q_{n}^{(c)}$ implements a queue of pointers to packets in node $n$ destined for node $c$. For each output port, let $Z_{n}^{(j)}$ denote the output queue in node $n$ that stores pointers to packets waiting at output $j$. With a slight abuse of notation, let $Z_{h}^{(j)}(t)$ also represent the current backlog of packets waiting at output $j$.

When a router receives a packet, it is linked to the tail of its corresponding per-destination queue and an output port queue. For O-BP, the choice of output port queue corresponds to the shortestpath output as determined by OSPF. For E-BP, one shortest-path output port is selected with equal probability. When a router is in Phase I, a packet will depart from the head of each output port queue, on the condition that the differential backlog for the commodity that this packet belongs to is positive, in which case the corresponding entry for that packet in the per-destination queue will be removed. Otherwise, if the differential backlog for the commodity that the packet at the head of an output port queue is non-positive, no packet will be moved. This ensures that our algorithms will never move a packet if the differential backlog is non-positive no matter whether the router is in Phase I or Phase II, which is required later in Section 5 for the throughput optimality proof.

When a router is in Phase II, the router will use the per-destination queue information of its neighbors to compute the backpressure weight, then choose the packet from the per-destination queue with the maximum positive weight, and forward it to its neighbor accordingly, and the corresponding entry in the output port queue will also be removed.

More specifically, the transition criteria for O-BP and E-BP are as follows:

- O-BP and E-BP: Let $H_{\max }$ be the maximum backlog that any output port queue $Z_{n}^{(j)}$ can have for a router to stay in Phase I. Whenever $Z_{n}^{(j)}(t)>H_{\max }$ for any output $j$, the entire router switches over to Phase II. Whenever $Z_{n}^{(j)}(t) \leq(1-\epsilon) H_{\text {max }}$ is satisfied for all outputs, the entire router returns back to Phase I. $\epsilon$ can be chosen to provide a safe margin between the transition thresholds to prevent a router from transitioning back and forth frequently between the two phases ( $\epsilon$ can be any number in $[0,1)$; e.g., in the evaluation section, we use $\epsilon=0)$.

\section{THROUGHPUT OPTIMALITY}

In this section, we use fluid models to prove that the L-BP, A-BP, O-BP, and E-BP algorithms are all throughput optimal.

\subsection{Modeling and Assumptions}

At each timeslot, each node needs to make a schedule to transmit data in the network. Let $\mathcal{S}$ be the set of all possible schedules. Each schedule $\beta=\left(\beta_{a b}^{(c)}: a, b, c=1, \ldots, N\right) \in \mathcal{S}$ is a vector in $\mathbb{Z}^{N \times N \times N}$, where $\beta_{a b}^{(c)}$ gives the amount of commodity $c$ data sent from node $a$ to node $b$ via link $(a, b)$ under schedule $\beta$. It is assumed that $\beta_{c n}^{(c)}=0$ for all $\beta \in \mathcal{S}, c=1, \ldots, N$ and $n=1, \ldots, N$. It is also assumed that $\mathcal{S}$ is monotone in the following sense: If $\beta \in \mathbb{Z}^{N \times N \times N}$ and there exists $\beta^{\prime} \in \mathcal{S}$ 
such that $\beta \leq \beta^{\prime}$, then $\beta \in \mathcal{S}$. This is because if $\beta^{\prime}$ is a valid schedule and we decrease the amount of data sent in some of the links, then the resulted schedule must also be a valid one.

We define $\mathcal{S}(t) \subset \mathcal{S}$ as the set of valid schedules given the scheduling strategy and the systems status at timeslot $t$. We assume that $\mathcal{S}(t)$ maintains the monotonicity of $\mathcal{S}$, i.e., $\beta \in \mathcal{S}(t)$ if there exists $\beta^{\prime} \in \mathcal{S}(t)$ such that $\beta \leq \beta^{\prime}$. A necessary constraint on $\mathcal{S}(t)$ is that we must have

$$
\sum_{b=1}^{N} \beta_{n b}^{(c)} \leq Q_{n}^{(c)}(t)
$$

for all $\beta \in \mathcal{S}(t)$ and $n, c=1, \ldots, N$. For each schedule $\beta \in \mathcal{S}$, we define a "collapsed" schedule $\gamma(\beta)=\left(\gamma_{n}^{(c)}(\beta): n, c=1, \ldots, N\right) \in \mathbb{Z}^{N \times N}$, where $\gamma_{n}^{(c)}(\beta)=\sum_{b=1}^{N} \beta_{n b}^{(c)}-\sum_{a=1}^{N} \beta_{a n}^{(c)}$ is the speed that schedule $\beta$ empties the backlog in queue $Q_{n}^{(c)}$. Let $\Gamma_{\mathcal{S}} \subset \mathbb{Z}^{N \times N}$ be the set of all possible collapsed schedules given $\mathcal{S}$. Let $<\Gamma_{\mathcal{S}}>$ be the convex hull of $\Gamma_{\mathcal{S}}$. Assume each link $(a, b), a, b=1, \ldots, N$, has a finite maximal transmission speed, i.e., $\exists R \geq 0$ such that $\beta_{a b}^{(c)} \leq R$ for all $\beta \in \mathcal{S}$ and $a, b, c=$ $1, \ldots, N$. Then both $\mathcal{S}$ and $\Gamma_{\mathcal{S}}$ are finite sets.

To analyze the stability of our schemes, we first define a family of generalized max-weighted scheduling schemes as follows. Define the weight $W(\beta, Q(t))$ of schedule $\beta$ given queue length $Q(t)$ as

$$
W(\beta, Q(t)) \triangleq \sum_{a=1}^{N} \sum_{b=1}^{N} \sum_{c=1}^{N} \beta_{a b}^{(c)}\left[Q_{a}^{(c)}(t)-Q_{b}^{(c)}(t)\right] .
$$

At each timeslot, a schedule $\beta \in \mathcal{S}$ that solves the following optimization problem will be selected for activation:

$$
\begin{array}{ll}
\max _{\beta} & W(\beta, Q(t)) \\
\text { s.t. } & \beta \in \mathcal{S}(t) .
\end{array}
$$

The baseline (original) backpressure scheme, our modified route-expanding and semi-oblivious BP schemes all belong to this family of generalized max-weighted scheduling schemes. The only difference among these schemes is the definition of the valid schedule set $\mathcal{S}(t)$ at each timeslot.

Let $\mathcal{S}^{B P}(t), \mathcal{S}^{L}(t), \mathcal{S}^{A}(t), \mathcal{S}^{O}(t), \mathcal{S}^{E}(t)$ be the set of valid schedules at timeslot $t$ for the baseline backpressure policy, the L-BP policy, the A-BP policy, the O-BP policy, and the E-BP policy, respectively.

We have

$$
\begin{aligned}
& \mathcal{S}^{B P}(t)=\left\{\beta \in \mathcal{S} \mid \sum_{b=1}^{N} \beta_{n b}^{(c)} \leq Q_{n}^{(c)}(t), \forall n, c=1, \ldots, N\right\} \\
& \mathcal{S}^{L}(t) \subseteq \mathcal{S}^{B P}(t) \\
& \mathcal{S}^{A}(t) \subseteq \mathcal{S}^{B P}(t) \\
& \mathcal{S}^{O}(t) \subseteq \mathcal{S}^{B P}(t) \\
& \mathcal{S}^{E}(t) \subseteq \mathcal{S}^{B P}(t) \\
& \mathcal{S}^{L}(t) \supseteq\left\{\beta \in \mathcal{S}^{B P}(t) \mid \beta_{n b}^{(c)}=0 \text { if } Q_{n}^{(c)}(t)<L_{\max },\right. \\
& \quad n, b, c=1, \ldots, N\},
\end{aligned}
$$




$$
\begin{array}{r}
\mathcal{S}^{A}(t) \supseteq\left\{\beta \in \mathcal{S}^{B P}(t) \mid \beta_{n b}^{(c)}=0 \text { if } E_{n}^{(c)}(t)<A_{\text {max }},\right. \\
n, b, c=1, \ldots, N\}, \\
\mathcal{S}^{O}(t) \supseteq\left\{\beta \in \mathcal{S}^{B P}(t) \mid \beta_{n b}^{(c)}=0 \text { if } Z_{n}^{(j)}(t)<H_{\text {max }},\right. \\
\left.n, b, c=1, \ldots, N, j=1, \ldots, M_{n}\right\}, \\
\mathcal{S}^{E}(t) \supseteq\left\{\beta \in \mathcal{S}^{B P}(t) \mid \beta_{n b}^{(c)}=0 \text { if } Z_{n}^{(j)}(t)<H_{\max },\right. \\
\left.n, b, c=1, \ldots, N, j=1, \ldots, M_{n}\right\} .
\end{array}
$$

In Equation (7), $E_{n}^{(c)}(t)$ represents the age of the head packet at time $t$, which is the number of timeslots that the head packet has waited in $Q_{n}^{(c)}$. In Equations (8) and (9), $M_{n}$ is the number of nodes neighbor to node $n$.

Let $T_{\beta}(t), \beta \in \mathcal{S}$, be the cumulative number of time slots that schedule $\beta$ was employed by timeslot $t$. From Equation (5), we have

$$
D_{a b}^{(c)}(t)=\sum_{\beta \in \mathcal{S}} \sum_{\ell=1}^{t} \beta_{a b}^{(c)} \cdot\left(T_{\beta}(\ell)-T_{\beta}(\ell-1)\right) .
$$

To formulate the fluid model, we extend the above discrete time functions to the continuous time domain. Specifically, for $t \in[0,+\infty)$, we define

$$
\begin{aligned}
& A_{n}^{(c)}(t)=A_{n}^{(c)}(\lfloor t\rfloor) \quad n, c=1, \ldots, N \\
& Q_{n}^{(c)}(t)=Q_{n}^{(c)}(\lfloor t\rfloor) \quad n, c=1, \ldots, N \\
& D_{a b}^{(c)}(t)=D_{a b}^{(c)}(\lfloor t\rfloor)+(t-\lfloor t\rfloor)\left(D_{a b}^{(c)}(\lceil t\rceil)-D_{a b}^{(c)}(\lfloor t\rfloor)\right), \\
& \quad a, b, c=1, \ldots, N \\
& T_{\beta}(t)=T_{\beta}(\lfloor t\rfloor)+(t-\lfloor t\rfloor)\left(T_{\beta}(\lceil t\rceil)-T_{\beta}(\lfloor t\rfloor)\right) \quad \beta \in \mathcal{S},
\end{aligned}
$$

where $\lfloor t\rfloor$ is the largest integer that is smaller than or equal to $t$ and $\lceil t\rceil$ is the smallest integer that is larger than or equal to $t$.

Assume the arrival process $A(t)$ satisfies a strong law of large numbers (SLLN), i.e., there exists a constant arrival rate matrix $\lambda=\left(\lambda_{n}^{(c)}: n, c=1, \ldots, N\right)$, such that, with probability 1 ,

$$
\lim _{t \rightarrow \infty} \frac{A_{n}^{(c)}(t)}{t}=\lambda_{n}^{(c)} \quad \forall n, c=1, \ldots, N .
$$

Without loss of generality, we assume that $\lambda_{c}^{(c)}=0$ for all $c=1, \ldots, N$ for simplicity.

\subsection{Basic Fluid Model Equations}

We now investigate the stochastic process $(Q(t), D(t), T(t))$, where

$$
\begin{aligned}
& Q(t) \triangleq\left\{Q_{n}^{(c)}(t) \mid n, c=1,2, \ldots, N\right\} \\
& D(t) \triangleq\left\{D_{a b}^{(c)}(t) \mid a, b, c=1,2, \ldots, N\right\} \\
& T(t) \triangleq\left\{T_{\beta}(t) \mid \beta \in \mathcal{S}\right\} .
\end{aligned}
$$


Let $(\Omega, \mathcal{F}, \mathbb{P})$ be the probability space that this stochastic process is defined on, where $\Omega$ is the sample space, $\mathcal{F}$ is a $\sigma$-field on $\Omega$, and $\mathbb{P}$ is the probability measure on $(\Omega, \mathcal{F})$. We shall sometimes use the notations $Q(\cdot, \omega), D(\cdot, \omega)$, and $T(\cdot, \omega)$ to explicitly denote the dependency on the sample path $\omega \in \Omega$.

Now, for each $r>0$, we define fluid scaled processes

$$
\left(\hat{Q}^{r}(t, \omega), \hat{D}^{r}(t, \omega), \hat{T}^{r}(t, \omega)\right) \triangleq \frac{1}{r}(Q(r t, \omega), D(r t, \omega), T(r t, \omega)) .
$$

Proposition 1 (Fluid Model). For each sample path $\omega \in \Omega$ satisfying (11) and any sequence $\left\{r_{n}\right\}$ with $r_{n} \rightarrow \infty$, there exists a subsequence $\left\{r_{n_{k}}\right\}$ and continuous functions $(\hat{Q}, \hat{D}, \hat{T})$ with $\hat{Q}(0)=0$, such that

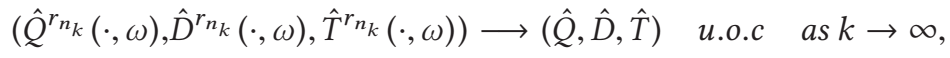

where the convergence is uniform on compact sets (u.o.c). The three-tuple $(\hat{Q}, \hat{D}, \hat{T})$ is said to be a fluid limit path of the system. It satisfies the following fluid model equations:

$$
\begin{aligned}
& \hat{Q}_{n}^{(c)}(t)=\lambda_{n}^{(c)} t+\sum_{a=1}^{N} \hat{D}_{a n}^{(c)}(t)-\sum_{b=1}^{N} \hat{D}_{n b}^{(c)}(t) \\
& n, c=1, \ldots, N \text { and } n \neq c, \\
& \hat{Q}_{c}^{(c)}(t)=0 \\
& c=1, \ldots, N, \\
& \hat{D}_{a b}^{(c)}(t)=\sum_{\beta \in \mathcal{S}} \beta_{a b}^{(c)} \hat{T}_{\beta}(t) \quad a, b, c=1, \ldots, N, \\
& \sum_{\beta \in \mathcal{S}} \hat{T}_{\beta}(t)=t, \\
& \hat{Q}_{n}^{(c)}(t) \geq 0 \quad n, c=1, \ldots, N \\
& \hat{T}_{\beta}(0)=0, \quad \hat{T}_{\beta}(\cdot) \text { is non-decreasing } \quad \beta \in \mathcal{S} \\
& \hat{T}_{\beta}(t)-\hat{T}_{\beta}(s) \leq t-s \text { for } 0 \leq s<t \quad \beta \in \mathcal{S} .
\end{aligned}
$$

The proof of Proposition 1 is somewhat standard. We refer the reader to Reference [7].

\subsection{Main Results}

We first give the formal definition of throughput optimilities.

Definition 1 (Rate Stability). We say the system is rate stable, if with probability 1 ,

$$
\lim _{t \rightarrow \infty} \frac{\sum_{n=1}^{N} D_{n c}^{(c)}(t)}{t}=\sum_{n=1}^{N} \lambda_{n}^{(c)} \quad c=1, \ldots, N
$$

for any arrival process satisfying Equation (11).

Note that the left-hand side of Equation (17) is actually the long-run average rate of commodity $c$ packets that depart from the network, while the right-hand side is the long-run average rate of commodity $c$ packets that arrive to the network. In other words, the system is guaranteed to achieve $100 \%$ throughput whenever it is rate stable.

Definition 2 (Throughput Optimal). We say an arrival process $A(t)$ is admissible if its arrival rate matrix $\lambda$ belongs to $\left\langle\Gamma_{\mathcal{S}}\right\rangle$. The system is said to be throughput optimal if it is rate stable under any admissible arrival process.

The main results of this section are stated as follows. 
THEOREM 1. The system is throughput optimal when working under the L-BP policy.

THEOREM 2. The system is throughput optimal when working under the A-BP policy.

Theorem 3. The system is throughput optimal when working under the O-BP or E-BP policy.

We will give the proof of these results using fluid model in the rest of this section. More specifically, we'll first prove that their fluid model is weakly stable as defined in Definition 3 and the stability of the original system is then guaranteed by Proposition 2. The detailed proof of Proposition 2 is provided in Appendix A.

Definition 3 (Weak Fluid Stability). The fluid model is said to be weakly stable if for each fluid limit path with $\hat{Q}(0)=0$ we have $\hat{Q}(t)=0$ for all $t \geq 0$.

Proposition 2. For a given arrival rate matrix $\lambda$, the system is rate stable if the corresponding fluid model is weakly stable.

\subsection{Proof of Theorems 1-3}

We first present a lemma that will be used in the proof of Theorems 1-3.

Lemma 1. The fluid model defined in Proposition 1 is weakly stable if each of the fluid limit paths satisfies the following fluid equation:

$$
\text { For each } \beta \in \mathcal{S}, \frac{d}{d t} \hat{T}_{\beta}(t)=0 \text { if } W(\beta, \hat{Q}(t))<\max _{\alpha \in \mathcal{S}} W(\alpha, \hat{Q}(t))
$$

The intuition behind Lemma 1 is that a scheduling policy is throughput optimal if, when the system backlog is very large, the weight of the schedule it generates is roughly the same with the weight of the schedule generated by the baseline backpressure policy. The proof of Lemma 1 is similar to the proof of Theorem 1 in Reference [7]. For completeness, we produce a full proof in Appendix B.

It is then sufficient to prove that each of the fluid limit paths satisfies Equation (18) if the system works under the L-BP, A-BP, O-BP, or E-BP policy. Intuitively, this is true, because when the backlog in the system is very large, the total backlog (and the total weight of the schedule) in the system would be dominated by the Phase II queues (as the queue lengths of the Phase I queues are always bounded by a constant). In other words, the contribution of Phase I queues is negligible when calculating the weight of a schedule. However, as Phase II queues always execute the baseline backpressure policy in our schemes, the weight of the generated schedule should be close to the weight of the schedule generated by the baseline backpressure policy. The formal proofs of Theorems 1-3 are similar to the proof of Lemma 4 in Reference [8]. For completeness, we produce a full proof in Appendices C-E.

\section{EVALUATIONS}

In this section, we present evaluations of our proposed route-expanding and semi-oblivious BP algorithms. To evaluate these algorithms, we focus on the adaptive routing problem for the wireline case. In particular, we present our evaluations using a real, large PoP-level backbone network, namely the Abilene[13] network. The Abilene network has been studied and discussed in the research literature. Its network topology, traffic dataset, and routing information are available in the public domain [28]. In the following, we first describe our experimental setup and then present our simulation results. 


\subsection{Experimental Setup}

The Abilene network is a public academic network in the U.S. with 12 nodes interconnected by OC192, 9.92 Gbits/s links. We use the traffic matrices obtained in Reference [28] in the experiments. Each traffic matrix consists of the demand rate of every source destination pair within five minutes. Therefore, these traffic matrices provide a snapshot of real total demand offerings between each source-destination pair in the Abilene network every 5 minutes. The actual dataset spans from March 1, 2004, to September 4, 2004. As the traffic matrices indicate, the Abilene network is underutilized. To demonstrate that our new backpressure-based algorithm improves delay performance while retaining optimal throughput, we selected the traffic matrix with the highest traffic load and scaled it by different factors. We incrementally increased the scaling factor until the resulting arrival rates exceed the network's stability region. Then we normalized that largest scaling factor.

We implemented our simulator in $\mathrm{C}++$. Traffic generation follows a Bernoulli arrival process with probability

$$
p=\frac{\text { traffic demand }}{\text { link capacity }}
$$

We assume $\mu_{a b}(t)=1$. That is, at each timeslot, at most one packet may be transmitted over each link. The end-to-end delay is measured by the time period from the timeslot when a packet enters the network by the traffic generation function to the timeslot when the packet arrives at its destination and thus leaves the network. To get reliable results, the simulation time should be long enough for the network to reach a steady state. In particular, for each scaling factor, we simulated 40 million timeslots, of which the first 20 million timeslots serve as a warm-up phase. We then collect 200 data points by sampling every 100,000 timeslots for the remaining 20 million timeslots. Finally, we average over these 200 data points for the results presented in this section for each scaling factor.

\subsection{Experimental Results}

6.2.1 Delay Performance. In this section, we present and compare simulaton results for endto-end delay performance for the original backpressure algorithm (BP), Open-Shortest-Path-First (OSPF) routing, Equal-Cost Multi-Path (ECMP) routing, Shortest-path backpressure (SPBP) routing, the L-BP and A-BP route-expanding $\mathrm{BP}$ routing algorithms, and the $\mathrm{O}-\mathrm{BP}$ and $\mathrm{E}-\mathrm{BP}$ semioblivious BP routing algorithms. The results are shown in Figure 2, Figure 3, and Figure 4. From these results, we can observe the following:

- Original BP: We observe in Figure 2 that under the original backpressure algorithm, the delay first decreases and then increases with increasing traffic loads. This phenomenon validates that the original backpressure algorithm incurs large delays under light or moderate traffic loads, because packets may explore unnecessarily long paths.

- OSPF and ECMP: From Figure 2(a), we observe that OSPF and ECMP have low average end-to-end delays compared with the original backpressure algorithm. However, they both saturate the network early, achieving only about $72 \%$ of the achievable throughput.

- SPBP: Figure 2(b) illustrates that although SPBP also achieves low average end-to-end delays, it is still not throughput optimal, because it saturates the network early, achieving similarly only about $72 \%$ of the achievable throughput. This is to be expected, since reducing routing choices shrinks the network stability region.

- L-BP: Figure 3(a) shows that L-BP route-expanding backpressure algorithm can achieve the same optimal throughput as the original backpressure algorithm and how the choices of the threshold $L_{\max }$ impact the delay performance. As expected, when $L_{\max }=0$, the delay 


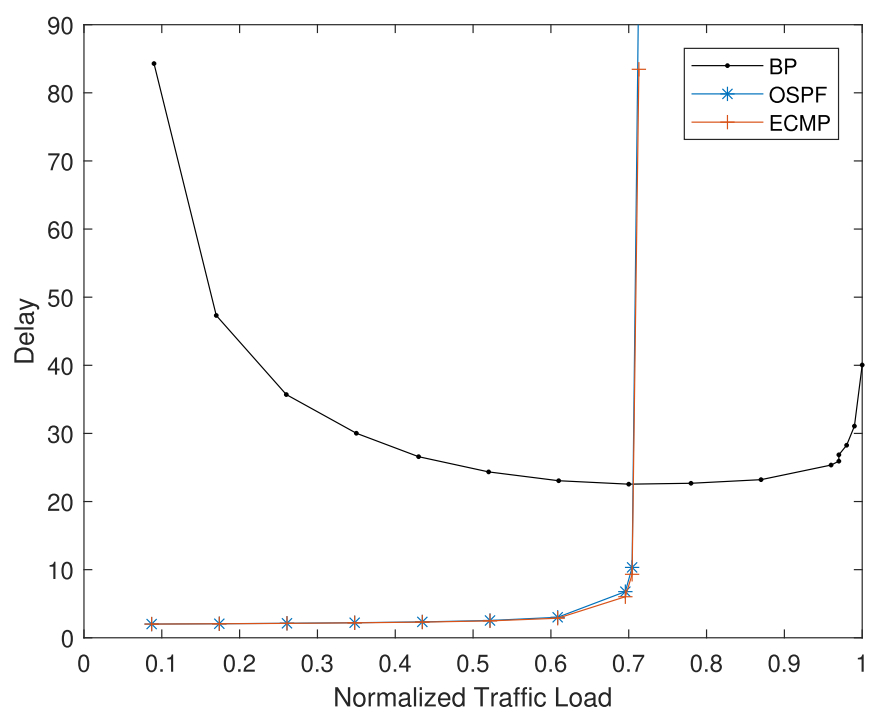

(a) BP vs. OSPF/ECMP

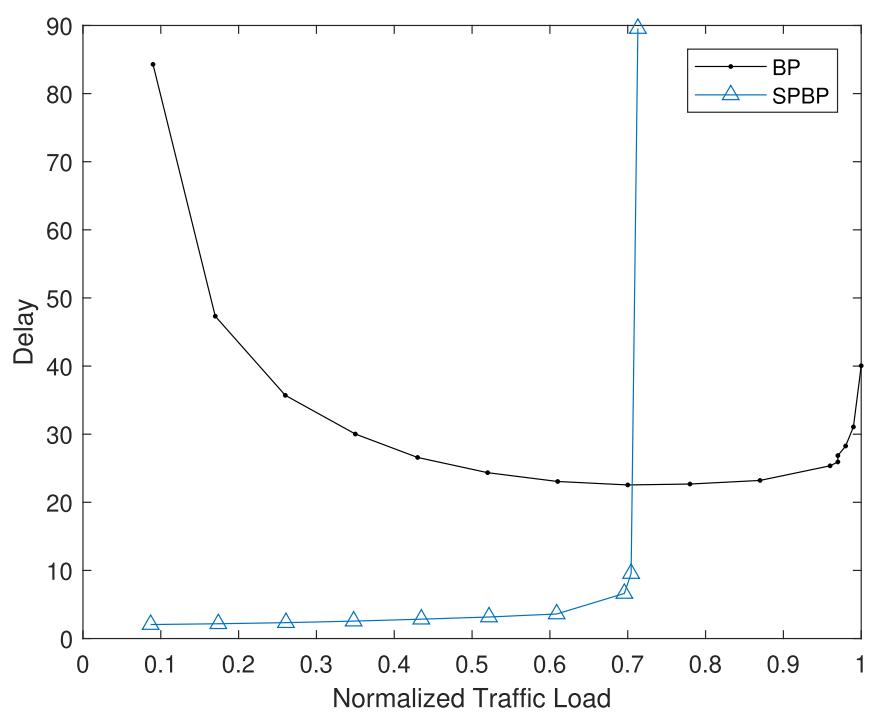

(b) BP vs. SPBP

Fig. 2. Delay comparison under different traffic loads for shortest-path routing.

performance is exactly the same as the original backpressure algorithm, because all perdestination queues are always in Phase II. Our experiments show that as long as $L_{\max } \geq 1$, the L-BP algorithm is able to reduce the delay by more than $80 \%$ compared with the original backpressure algorithm when the traffic load is light or moderate. To obtain better delay performance, $L_{\max }$ should be carefully chosen and tuned. Intuitively, $L_{\max }$ should not be too big, as a big $L_{\max }$ may impede the entrance into Phase II and the accumulated packets may have larger queuing delays than those in a smaller $L_{\max }$ setting. For example, as illustrated in Figure 3(a), the delay for $L_{\max }=10$ increases significantly at higher traffic loads, because 


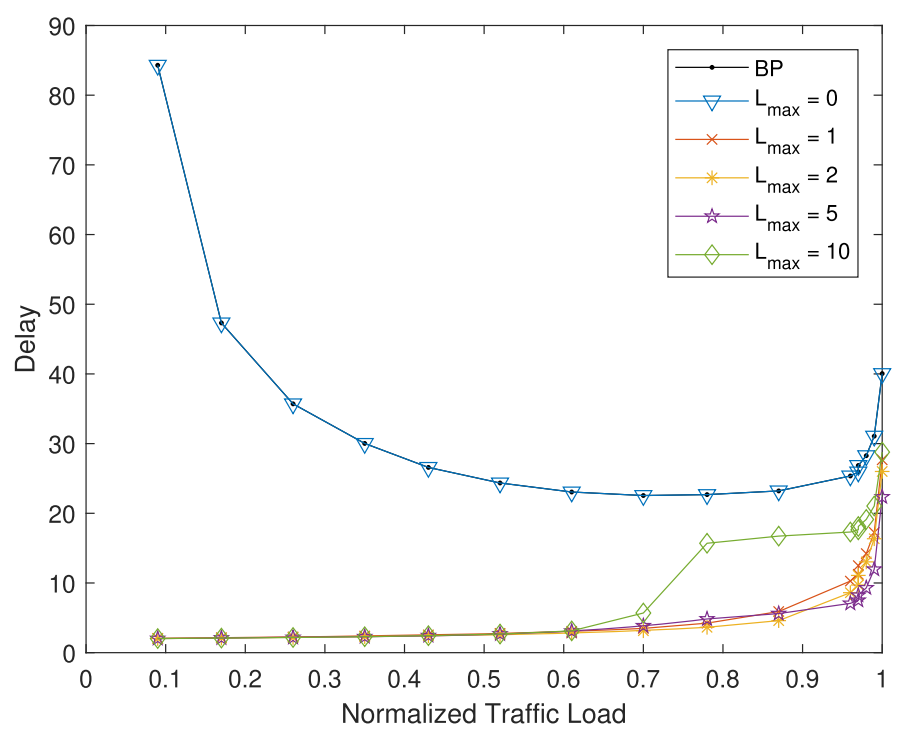

(a) BP vs. L-BP

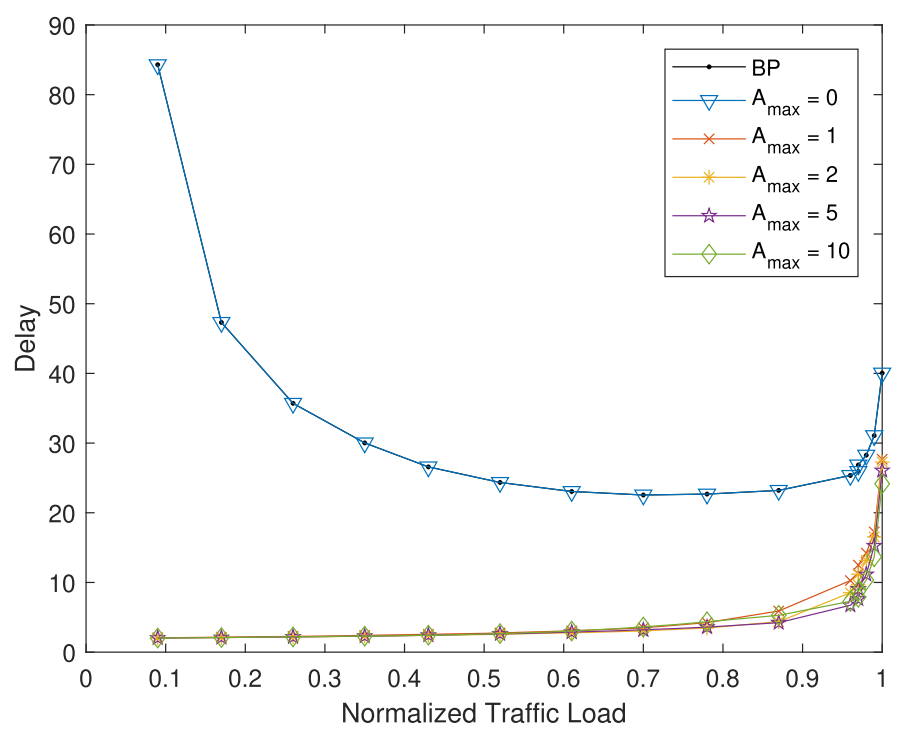

(b) BP vs. A-BP

Fig. 3. Delay comparison under different traffic loads for route-expanding backpressure routing.

those congested per-destination queues do not enter Phase II in time. However, if $L_{\max }$ is too small, then it would be too easy for the per-destination queues to enter Phase II and route the packets unnecessarily to longer paths.

- A-BP: Figure 3(b) shows that A-BP route-expanding backpressure algorithm can achieve the same optimal throughput as the original backpressure algorithm and how the choices of the threshold $A_{\max }$ impact the delay performance. Similarly to L-BP, when $A_{\max }=0$, the delay performance is exactly the same as the original backpressure algorithm, because all 


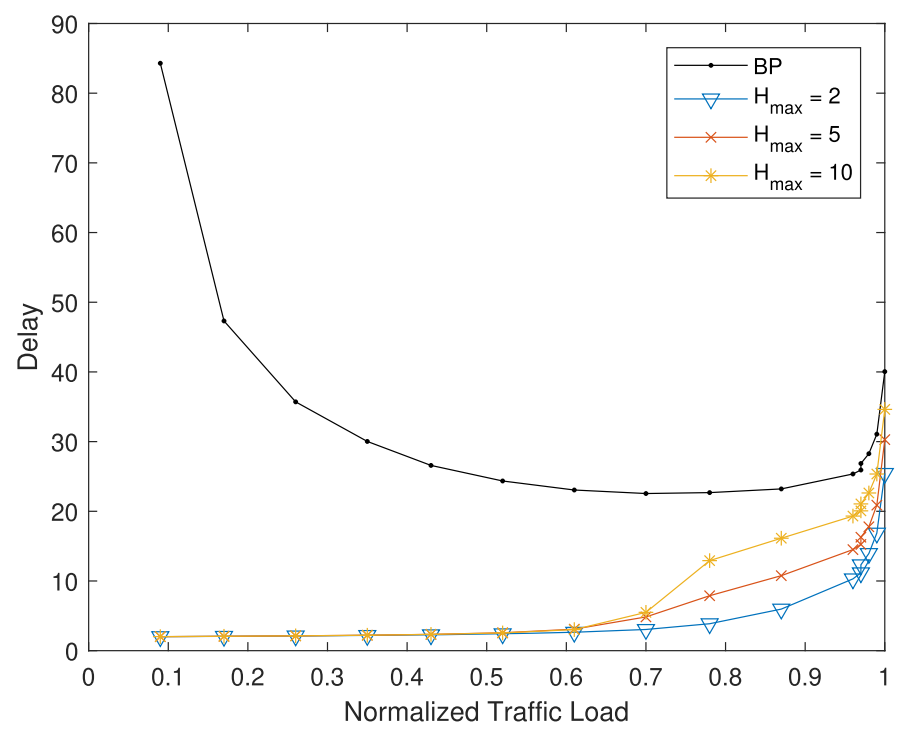

(a) BP vs. O-BP

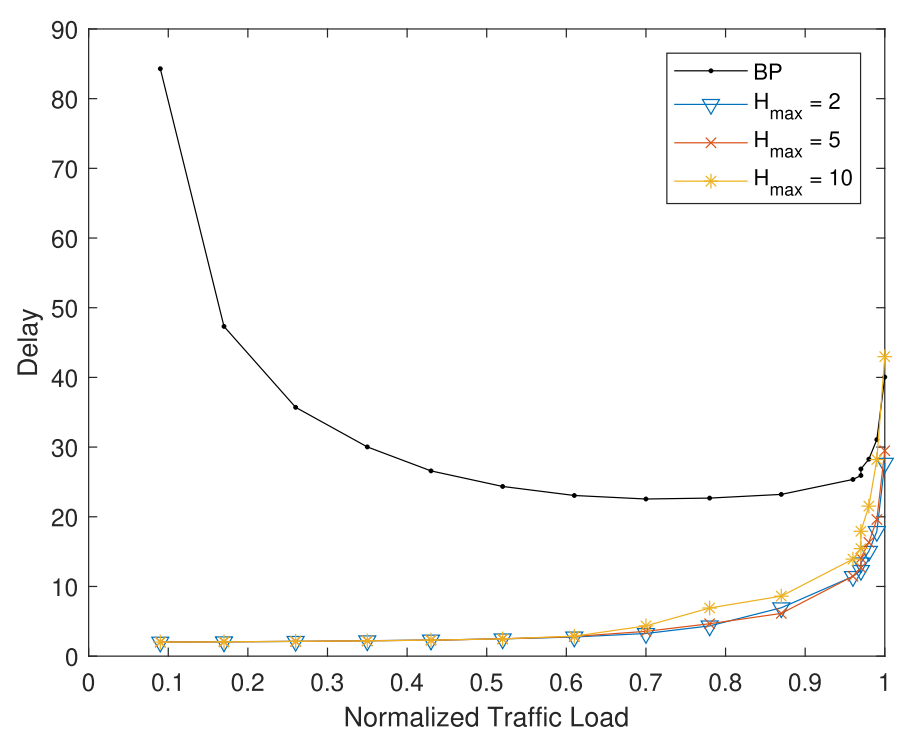

(b) BP vs. E-BP

Fig. 4. Delay comparison under different traffic loads for semi-oblivious backpressure routing.

per-destination queues are always in Phase II. In addition, our experiments show that as long as $A_{\max } \geq 1$, the A-BP algorithm is able to reduce the delay by more than $90 \%$ compared with the original backpressure algorithm when the traffic load is light or moderate. The parameter $A_{\max }$ should also be tuned carefully. For the same reason, $A_{\max }$ should not be too big, as a big $A_{\max }$ may prevent the queue from entering Phase II in time. Also, if the threshold is too small (e.g. $A_{\max }=1$ ), then it makes the queues too easy to enter Phase II. 
- O-BP and E-BP: Figure 4 shows that, similarly to L-BP and A-BP, the O-BP and E-BP semioblivious backpressure algorithms can also both achieve the same optimal throughput as the original backpressure algorithm. Our experiments show that when $H_{\max } \geq 2$, both O$\mathrm{BP}$ and E-BP are able to reduce the delay at light or moderate traffic dramatically. As before, $H_{\max }$ should be chosen carefully to achieve better performance. In general, smaller $H_{\max }$ is more favorable. In our experiments, $H_{\max }=2$ has the best delay performance for both $\mathrm{O}-\mathrm{BP}$ and E-BP algorithms.

6.2.2 Backlog Information Exchanges in L-BP and A-BP. In this section, we examine two metrics: the backlog information exchange frequency and the percentage of queues in the network that are in Phase II for L-BP and A-BP.

A backlog information exchange is recognized when an internal per-destination queue needs its neighbor's corresponding per-destination queue backlog information to compute the backpressure. For example, according to the original backpressure algorithm, if a node has three neighbors, then each internal per-destination queue has to know the backlog information from its three neighbors, and the corresponding differential backlogs need to be computed for these three neighbors. However, in our proposed algorithms, if a queue is in Phase I, then the backlog information only needs to be exchanged with a subset of neighbor nodes that are a part of some shortest path routes, and the differential backlog calculations only need to be computed with respect to these nodes.

It should be noted that, among the original $\mathrm{BP}$ algorithm, SPBP, L-BP, and A-BP, the original $\mathrm{BP}$ algorithm needs the maximum number of backlog information exchanges, which is the upper bound. SPBP needs the least, which is the lower bound. For the L-BP/A-BP algorithms, whether their backlog information exchange frequencies are closer to BP or SPBP depends on how many queues in L-BP/A-BP are in Phase II. If no queue in L-BP/A-BP is in Phase II, then L-BP/A-BP is the same as SPBP. If all queues in L-BP/A-BP are in Phase II, then L-BP/A-BP is the same as BP.

Compared with SPBP, which is not throughput optimal, L-BP/A-BP adaptively transitions perdestination queues to Phase II from Phase I as needed to achieve the optimal throughput. However, the more per-destination queues are transitioned into Phase II, the higher is the amount of backlog information that a node has to exchange with its neighbors. Figure 5 shows that the increase in backlog information exchange is very small, which is a small price to pay for optimal throughput.

In particular, Figure 5(a) shows the backlog information exchange frequency for BP, SPBP, L$\mathrm{BP}$, and $\mathrm{A}-\mathrm{BP}$ algorithms, under different traffic loads. The information exchange frequency is normalized to the number of backlog exchanges required by $\mathrm{BP}$, which has the highest frequency as $\mathrm{BP}$ requires information exchanges with all neighbors. However, SPBP has the lowest frequency as it only requires information exchanges with shortest-path neighbors; the normalized frequency for SPBP is about 0.45 . Surprisingly, L-BP and A-BP only require slightly more backlog information exchanges compared with SPBP, even at very high traffic loads. This means that, by adding a bit more backlog information exchanges, L-BP and A-BP can achieve optimal throughput, which is a much higher throughput than SPBP. In Figure 5(b), we can see an increasing number of queues are switched over to Phase II when the traffic load increases. For the same group of algorithms, $\mathrm{L}-\mathrm{BP}$ or A-BP, the smaller the threshold is, the more queues are switched over to Phase II as the the traffic load increases, because a smaller threshold makes the router easier to switch over the Phase II. Nevertheless, the increase is negligible if the threshold and algorithm is properly chosen. For example, the percentage of Phase II routers for L-BP $\left(L_{\max }=5\right)$, even under the highest traffic load, is still less than 3\%. For others, even under very high traffic loads, the percentages are still below $23 \%$. All of this translates to much lower computational requirements for calculating the necessary different backlogs. 


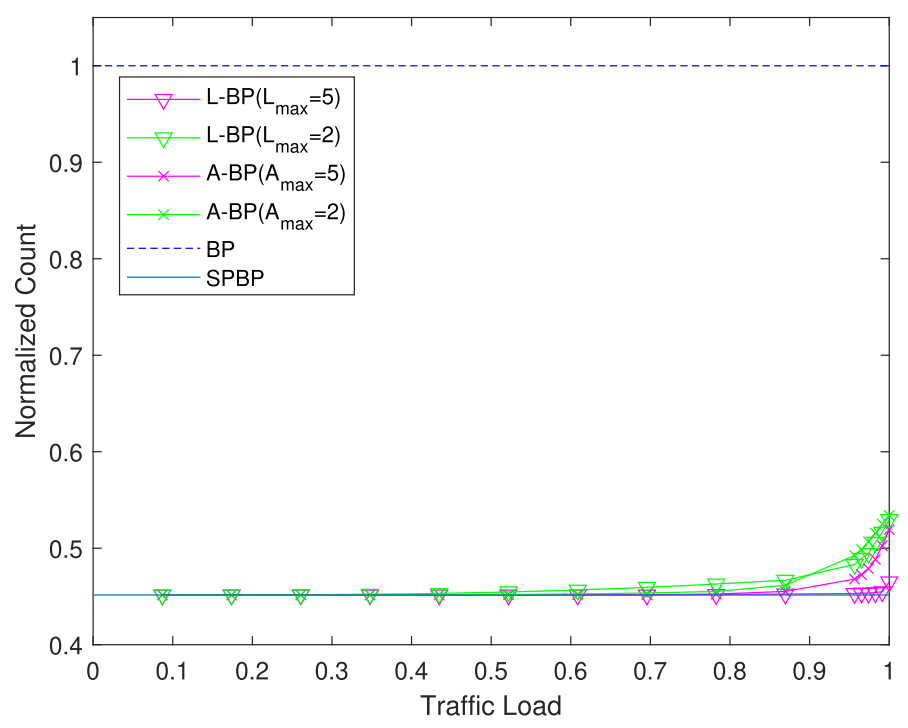

(a) Backlog information exchange frequency.

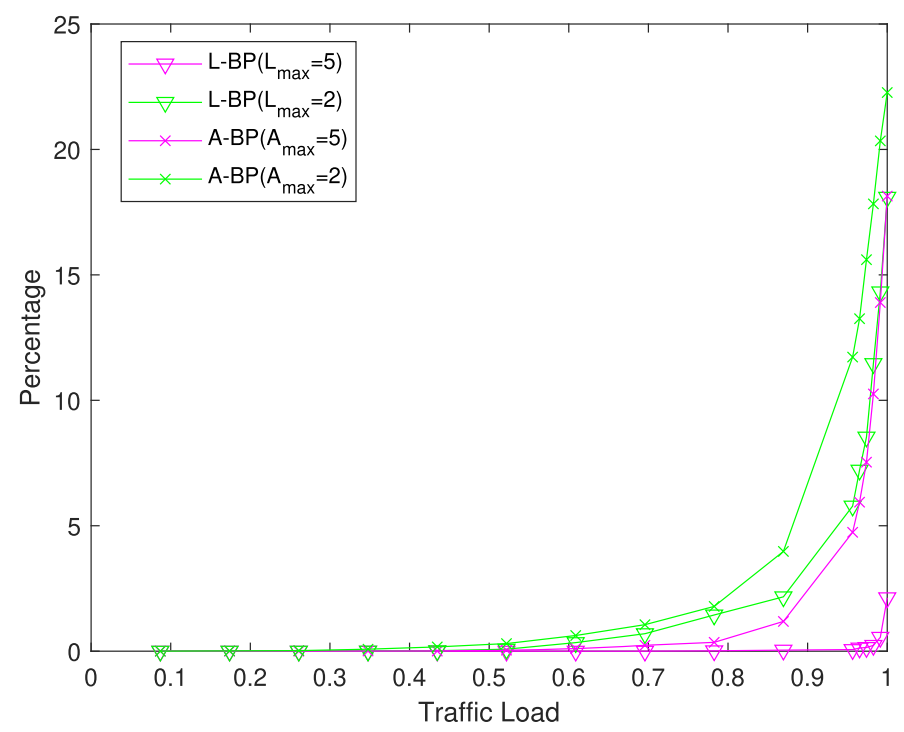

(b) Percentages of Phase II queues.

Fig. 5. Backlog information exchange frequency and percentages of Phase II queues for L-BP and A-BP.

Figure 6 and Figure 7 take the maximum traffic load (the traffic load we use in our experiments that is just about to saturate the network) as an example, and show the number of backlog information exchanges and the percentages of Phase II queues for L-BP and A-BP, respectively.

In every figure, we can see that a bigger threshold usually incurs less information exchanges, because fewer routers are switched over to Phase II. With a carefully chosen threshold, an algorithm may only require the similar amount of backlog information exchanges to SPBP algorithm. For example, in Figure 6(a), most data points from L-BP $\left(L_{\max }=5\right)$ for the backlog information 


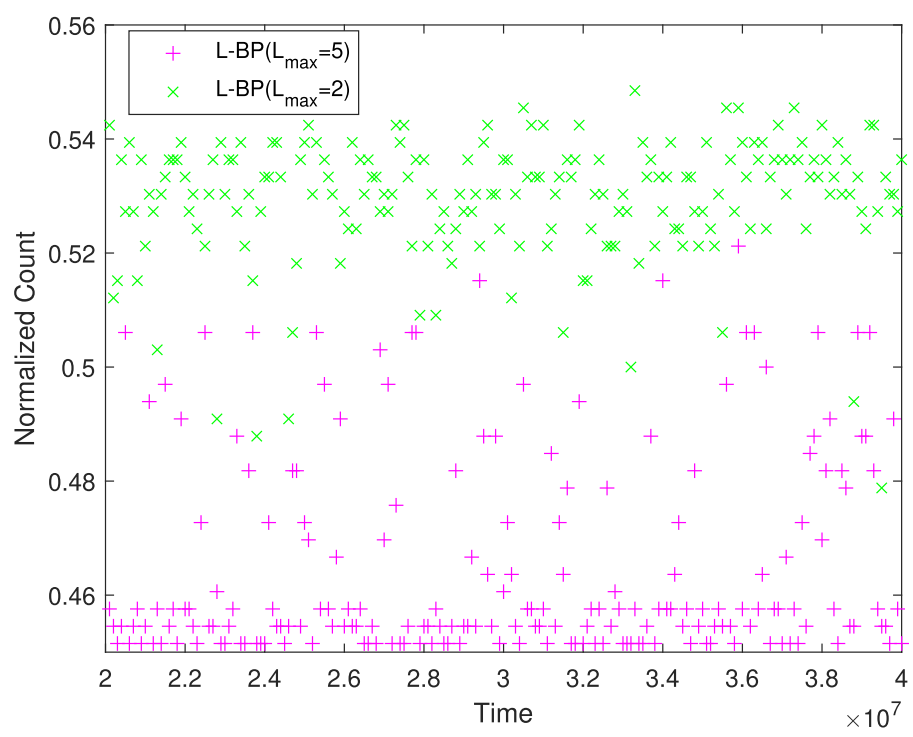

(a) Backlog information exchange frequency.

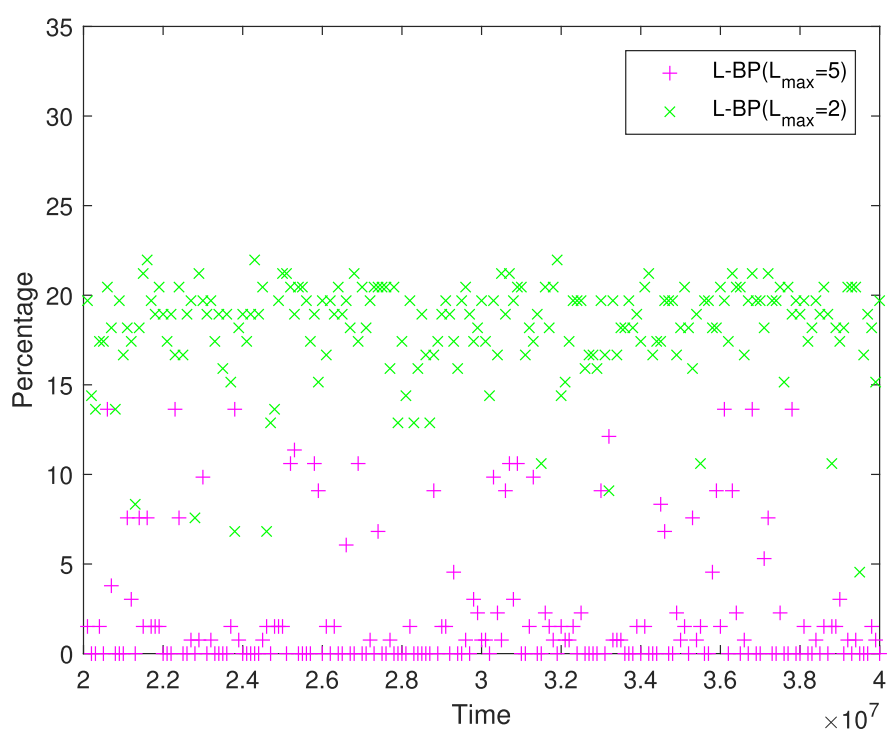

(b) Percentages of Phase II queues.

Fig. 6. Backlog information exchange frequency and percentages of Phase II queues for L-BP under maximum traffic load.

exchanges are between 0.45 and 0.46. Recall that the lower bound from SPBP for the normalized backlog information exchange frequency is about 0.45 . Therefore, Figure 6(a) shows that most of the time the backlog information exchange frequency of L-BP $\left(L_{\max }=5\right)$ is comparable to the SPBP algorithm. For other algorithms, as can be seen from Figure 6(a) and Figure 7(a), most of the data points are below 0.54 , which indicates that most of the time the frequency of the backlog information exchanges is also limited. 


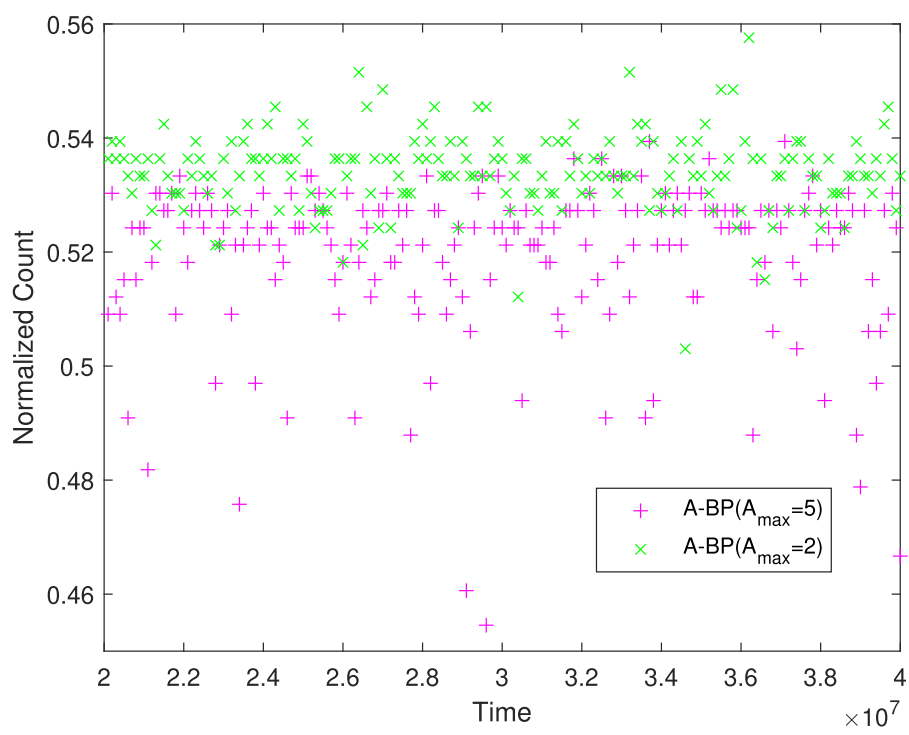

(a) Backlog information exchange frequency.

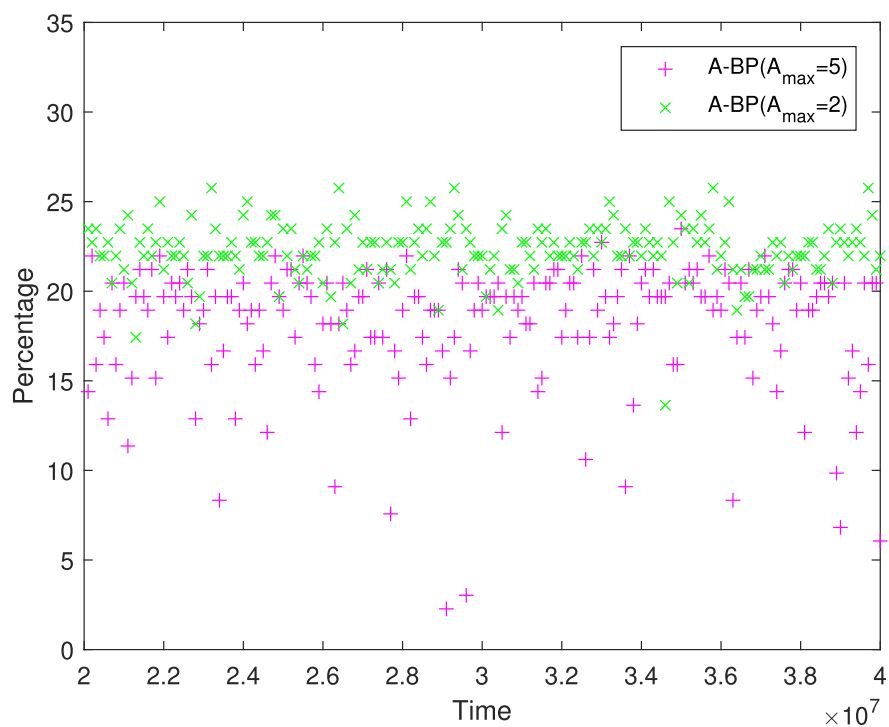

(b) Percentages of Phase II queues.

Fig. 7. Backlog information exchange frequency and percentages of Phase II queues for A-BP under maximum traffic load.

Figure 6(b) and Figure 7(b) show that most of the time only less than $25 \%$ queues are switched over to Phase II. This indicates that our algorithms are able to identify the most congested areas of the network, switch over those queues to Phase II, and keep the remaining queues working in Phase I. 


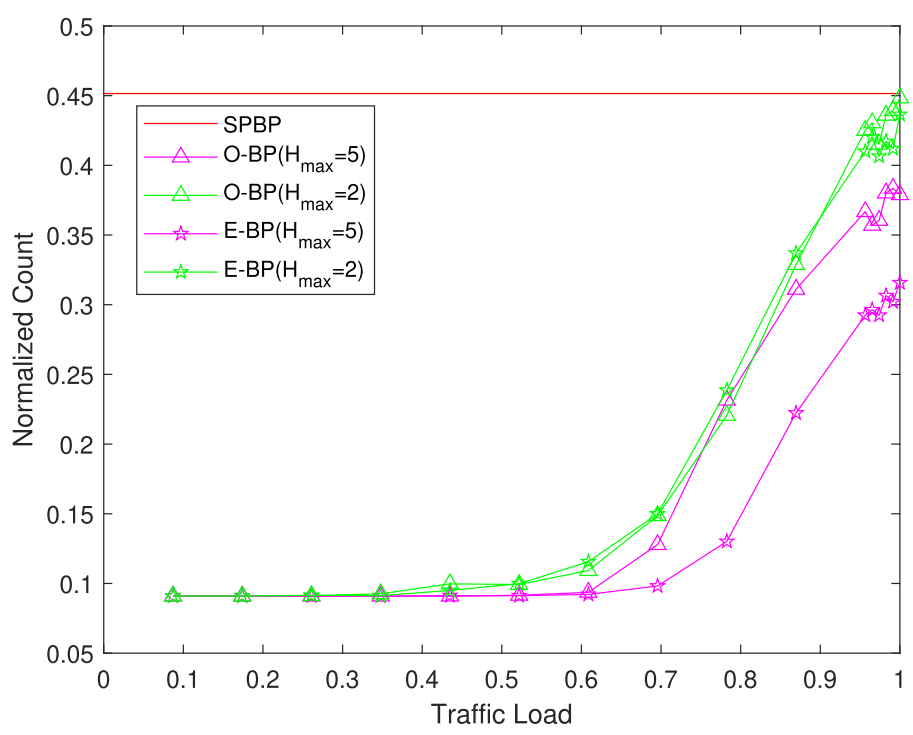

(a) Backlog information exchange frequency.

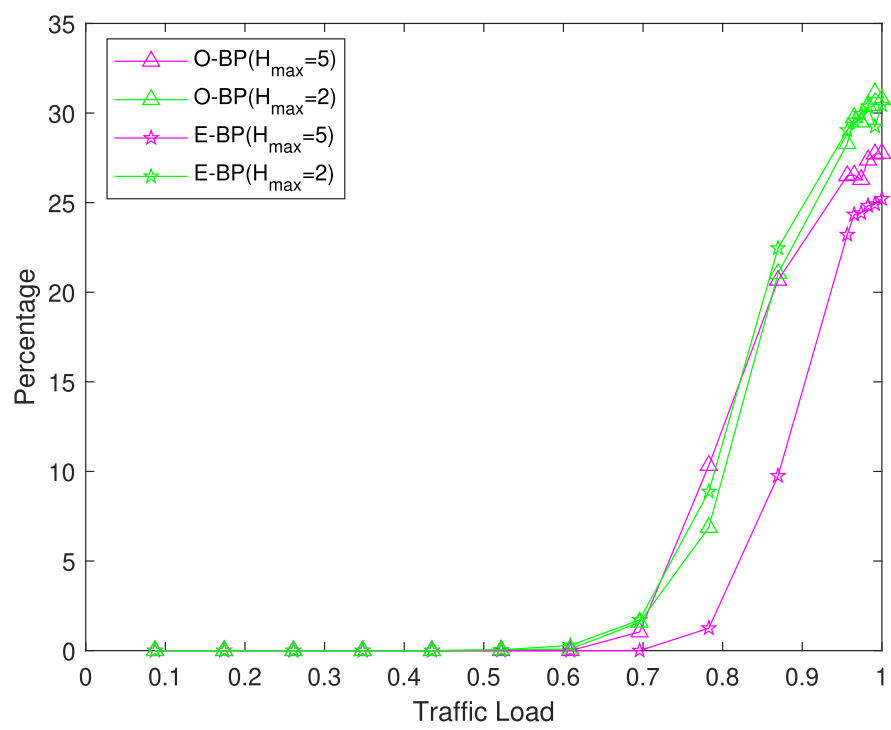

(b) Percentages of Phase II routers.

Fig. 8. Backlog information exchange frequency and percentages of Phase II routers for O-BP and E-BP.

6.2.3 Backlog Information Exchanges in $O-B P$ and $E-B P$. In this section, we examine the backlog information exchange frequency and the percentage of routers in the network that are in Phase II for the O-BP and E-BP semi-oblivious BP algorithms.

When a router is in Phase I, for each link, it only needs backlog information from the corresponding neighbor for a single commodity, which the packet at the head of the output port queue belongs to. In comparison, when a router is in Phase II, it needs the backlog information from 


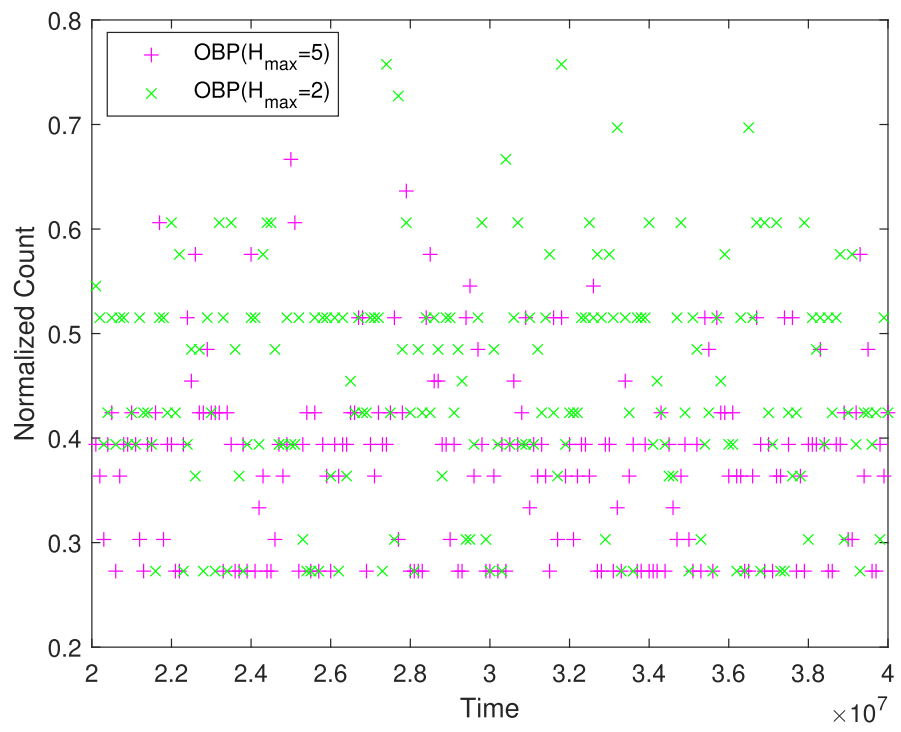

(a) Backlog information exchange frequency.

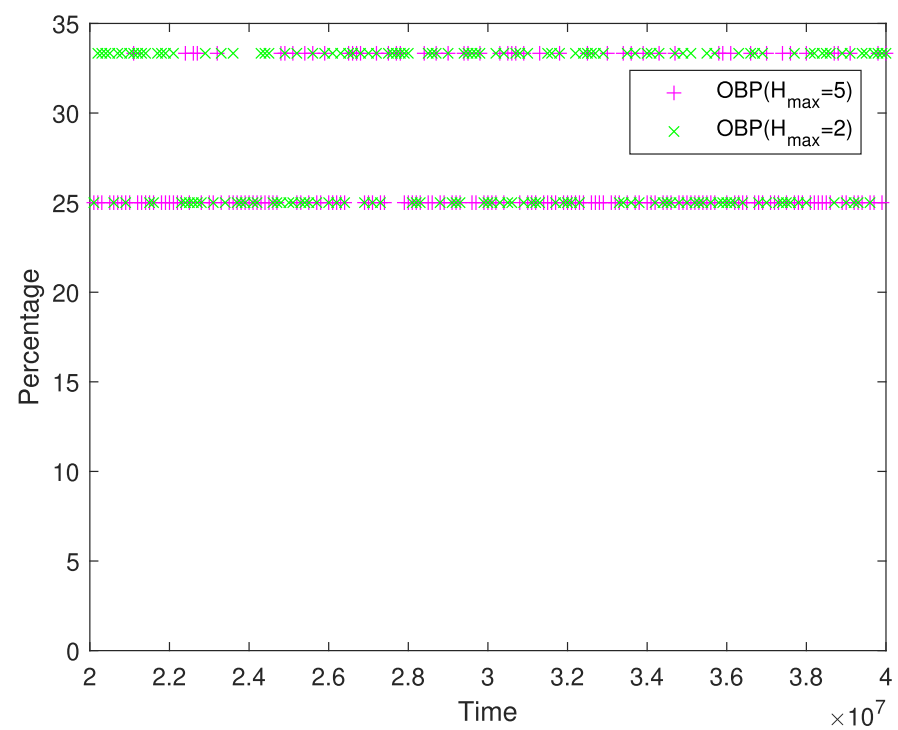

(b) Percentages of Phase II routers.

Fig. 9. Backlog information exchange frequency and percentages of Phase II routers for O-BP under maximum traffic load.

all its neighbors for all commodities. Figure 8(a) compares the backlog information exchange frequency among SPBP, O-BP and E-BP algorithms under different traffic loads. As we can see, O-BP and E-BP require substantially lower frequency of backlog information exchanges. This is because when the traffic load is not high, most routers are still in Phase I, and thus they only need very few information exchanges. Even when the traffic load becomes high, O-BP and E-BP still incur less 


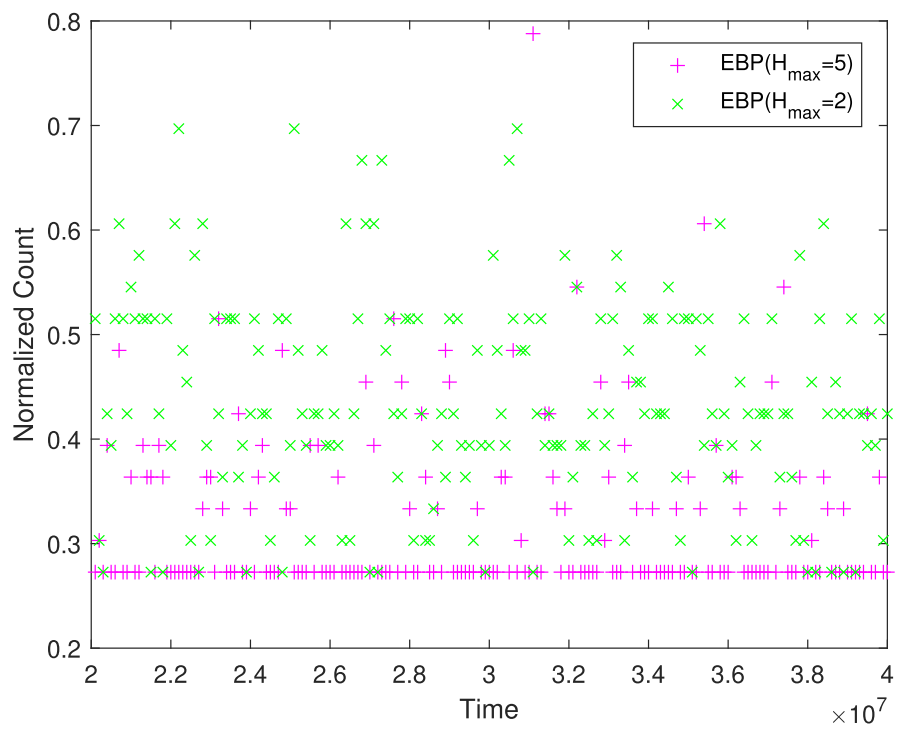

(a) Backlog information exchange frequency.

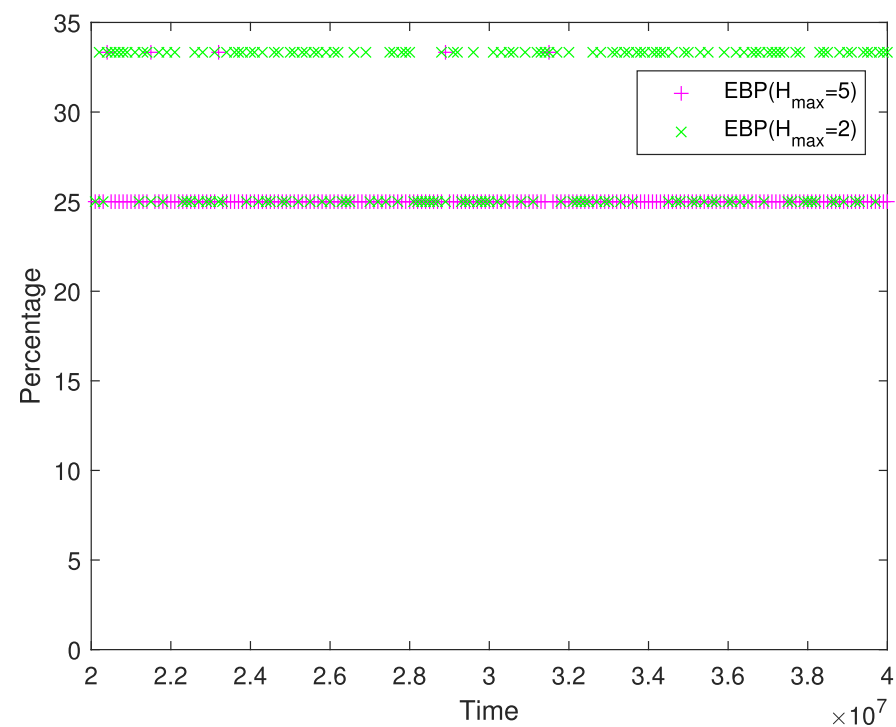

(b) Percentages of Phase II routers.

Fig. 10. Backlog information exchange frequency and percentages of Phase II routers for E-BP under maximum traffic load.

information exchanges than SPBP. This is because, as shown in Figure 8(b), a large percentage of the routers in the network still remain in Phase I, and these Phase I routers only need a small fraction of backlog information from their neighbors. Although the Phase II routers have to exchange backlog information with its neighbors for all commodities, the number of such Phase II routers is very small. As a result, $\mathrm{O}-\mathrm{BP}$ and $\mathrm{E}-\mathrm{BP}$ always require fewer information exchanges than SPBP for the entire range of traffic loads simulated. 


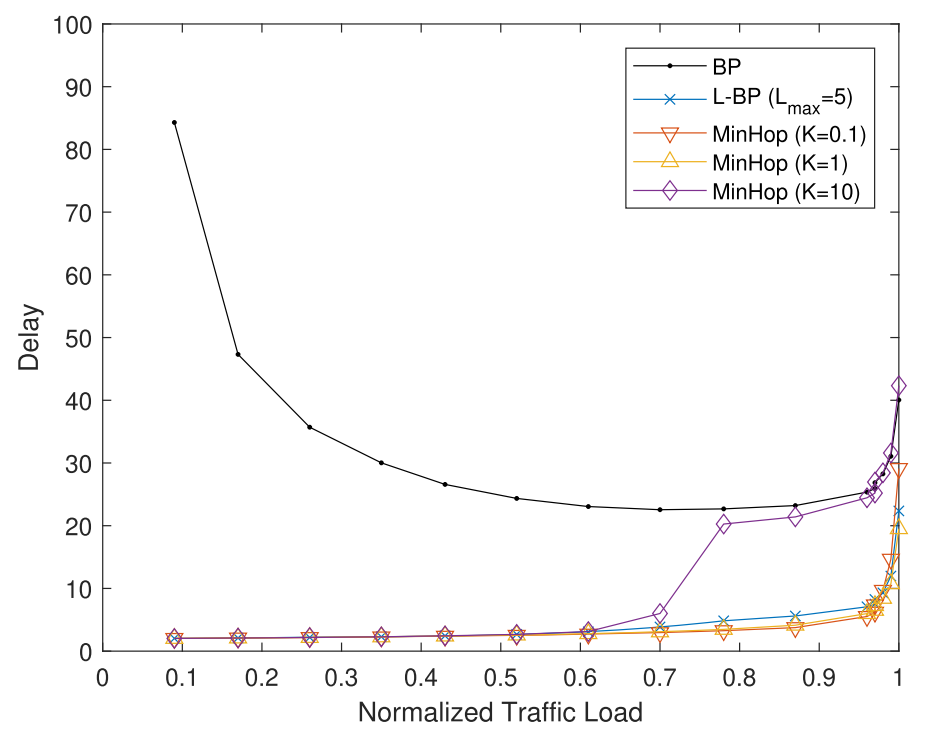

(a) Delay comparison with [26].

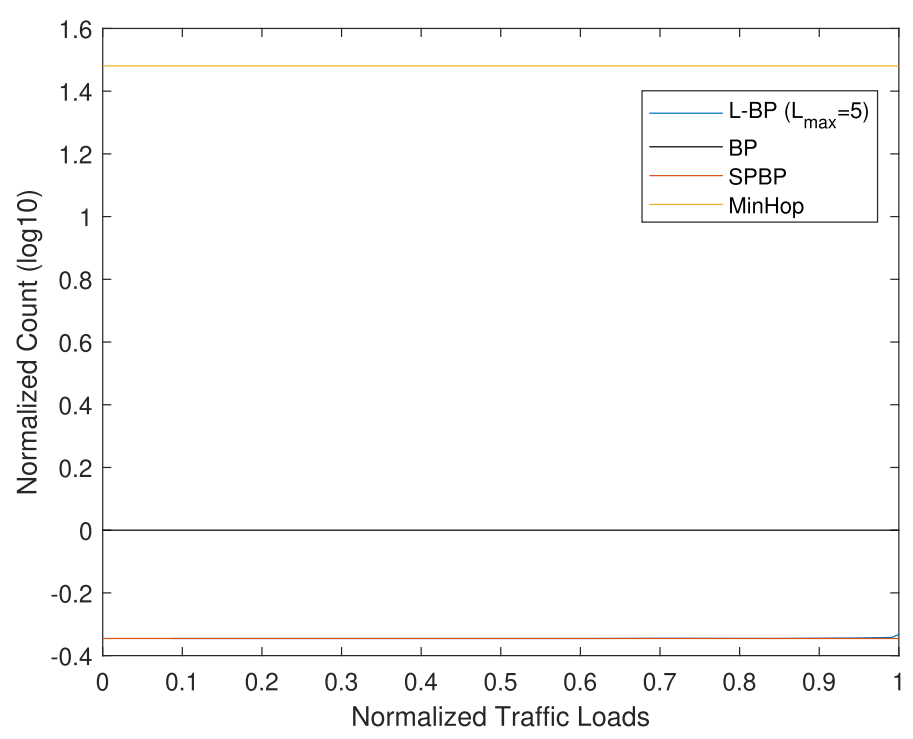

(b) Information exchanges comparison with [26].

Fig. 11. Compare with Reference [26] (referred here as "MinHop").

Figure 9(a) and Figure 10(a) show the amount of backlog information exchanges at the maximum traffic load for O-BP and E-BP, respectively. Similarly to L-BP and A-BP algorithms, a bigger threshold leads to less information exchanges, because more routers are still in Phase I. With a carefully chosen threshold, our algorithm can reduce the information exchanges dramatically. Take $H_{\max }=5$ as an example: Even under this maximum traffic load, the normalized amount the information exchanges of either of O-BP or E-BP rarely exceeds 0.45 , which is even less than the 
normalized frequency required by SPBP. This is because, as shown in Figure 9(b) and Figure 10(b), even when the traffic load is about to saturate the network, there are still about $65 \%$ to $75 \%$ routers that are in Phase I, which only need a small amount of backlog information from their neighbors. Besides, that the percentage is concentrated at a fixed number of values indicates that only some particular routers are congested in the network at this traffic load. Our algorithms are able to identify the congested areas in the network and only switch over those routers to Phase II.

6.2.4 Comparison with Prior Proposed Algorithms. We have implemented the MinHopalgorithm proposed in Reference [26] to compare with our proposed algorithms in Figure 11.

Figure 11(a) shows that our delay performance is comparable with the MinHop algorithm. The delay performance of the MinHop algorithm is sensitive to the choice of the parameter $K$. If $K$ is too big, then their algorithm will favor shortest paths. As long as the $K$ is not infinite, the throughput still remains optimal, but the delay can be extremely huge. However, if $K$ is too small, for example $K=0.1$, then the delay is also compromised, because it just favors shorter queues. As a result, $K$ plays an important role in improving delay performance, which is similar to what our $L_{\max }, A_{\max }$, and $H_{\max }$ do.

The main drawback of Reference [26] is its complexity. As can be seen from Figure 11(b), due to the huge number of queues that must be maintained per router, the amount of information exchanges is enormous. When compared with even the original backpressure algorithm, the amount of information exchanges required by MinHop [26] is 30 times higher, which in turn is far higher than what our algorithms require.

\section{CONCLUSION}

In this article, we proposed two new route-expanding backpressure-based adaptive routing algorithms, called L-BP and A-BP, that are based on the incremental expansion of routing choices in response to congestion at a node on a per-destination basis. We also proposed two new semioblivious backpressure-based adaptive routing algorithms, called $\mathrm{O}-\mathrm{BP}$ and E-BP, that are based on oblivious routing by default and incremental expansion to backpressure routing on a per-node basis. All variants are shown to be throughput optimal by means of fluid model analysis. Simulation results using actual traffic profiles on a public network demonstrate that our proposed algorithms indeed provide substantial improvements in delay performance. The simulation results further show that in practice, our approach dramatically reduces the amount of differential backlogs that has to be computed and the amount of backlog information that has to be exchanged, because routing choices are only incrementally expanded as needed.

\section{APPENDICES}

\section{A PROOF OF PROPOSITION 2}

Proof. From our assumptions in Section 5.1, we know that $\beta_{c n}^{(c)}=0$ for all $\beta \in \mathcal{S}$ and $n, c=$ $1, \ldots, N$. Then by Equation (15), we know that $\hat{D}_{c n}^{(c)}(t)=0$ for all $n, c=1, \ldots, N$. This equality will be used in justifying Equation (19) below.

Denote $\mathcal{N}=\{1, \ldots, N\}$ as the set of nodes in the network. From Equations (13) and (14), for each commodity $c$, we have

$$
\begin{aligned}
\sum_{n=1}^{N} \hat{Q}_{n}^{(c)}(t) & =\sum_{n \in \mathcal{N} \backslash\{c\}} \hat{Q}_{n}^{(c)}(t) \\
& =\sum_{n \in \mathcal{N} \backslash\{c\}}\left(\lambda_{n}^{(c)} t+\sum_{a=1}^{N} \hat{D}_{a n}^{(c)}(t)-\sum_{b=1}^{N} \hat{D}_{n b}^{(c)}(t)\right)
\end{aligned}
$$




$$
\begin{aligned}
= & \sum_{n \in \mathcal{N} \backslash\{c\}} \lambda_{n}^{(c)} t+\sum_{n \in \mathcal{N} \backslash\{c\}} \sum_{a=1}^{N} \hat{D}_{a n}^{(c)}(t) \\
& -\sum_{n \in \mathcal{N} \backslash\{c\}} \sum_{b=1}^{N} \hat{D}_{n b}^{(c)}(t) \\
= & \sum_{n=1}^{N} \lambda_{n}^{(c)} t+\sum_{n \in \mathcal{N} \backslash\{c\}} \sum_{a=1}^{N} \hat{D}_{a n}^{(c)}(t)-\sum_{n=1}^{N} \sum_{b=1}^{N} \hat{D}_{n b}^{(c)}(t), \\
= & \sum_{n=1}^{N} \lambda_{n}^{(c)} t-\sum_{c=1}^{N} \hat{D}_{n c}^{(c)}(t) .
\end{aligned}
$$

Equation (19) stands, because (1) $\lambda_{c}^{(c)}=0$ for $c=1, \ldots, N$ from our assumptions in Section 5.1 and (2) $\hat{D}_{c n}^{(c)}(t)=0$ for $n, c=1, \ldots, N$.

If the fluid model is weakly stable, by Definition 3, then we know that $\sum_{n=1}^{N} \hat{Q}_{n}^{(c)}(t)=0$ for all $t \geq 0$. Then by Equation (20), we have

$$
\sum_{c=1}^{N} \hat{D}_{n c}^{(c)}(t)=\sum_{n=1}^{N} \lambda_{n}^{(c)} t
$$

Thus

$$
\sum_{c=1}^{N} \hat{D}_{n c}^{(c), r}(t, \omega) \rightarrow \sum_{n=1}^{N} \lambda_{n}^{(c)} t \quad \text { u.o.c } \quad \text { as } r \rightarrow \infty .
$$

In particular, $\sum_{c=1}^{N} \hat{D}_{n c}^{(c), r}(1, \omega) \rightarrow \sum_{n=1}^{N} \lambda_{n}^{(c)}$ as $r \rightarrow \infty$ or

$$
\lim _{r \rightarrow \infty} \frac{\sum_{n=1}^{N} D_{n c}^{(c)}(r)}{r}=\sum_{n=1}^{N} \lambda_{n}^{(c)},
$$

which is actually the same as Equation (17), proving the proposition.

\section{B PROOF OF LEMMA 1}

Proof. The proof is similar to the proof of Theorem 1 in Reference [7]. For completeness, we produce a full proof here.

It is sufficient to show that the fluid model is weakly stable for any arrival rate matrix $\lambda$ that belongs to $<\Gamma_{\mathcal{S}}>$. Fix an arrival rate matrix $\lambda$ that belongs to $<\Gamma_{\mathcal{S}}>$. Suppose $(\hat{Q}, \hat{D}, \hat{T})$ is a fluid limit path with $|\hat{Q}(0)|=0$. Define a Lyapunov function

$$
f(t)=\sum_{n=1}^{N} \sum_{c=1}^{N}\left(\hat{Q}_{n}^{(c)}(t)\right)^{2} .
$$

We have $f(0)=0, f(t) \geq 0$, and $f(t)=0 \Leftrightarrow \hat{Q}(t)=0$ for all $t>0$. It is then sufficient to prove that such that $f(t)=0$ for all $t \geq 0$. 
Since $\lambda$ belongs to $\left\langle\Gamma_{\mathcal{S}}>\right.$, there exist constants $p_{\beta} \in[0,1], \beta \in \mathcal{S}$, such that

$$
\begin{aligned}
& \lambda_{n}^{(c)}=\sum_{\beta \in \mathcal{S}} p_{\beta} \cdot \gamma_{n}^{(c)}(\beta)=\sum_{\beta \in \mathcal{S}} p_{\beta}\left(\sum_{b=1}^{N} \beta_{n b}^{(c)}-\sum_{a=1}^{N} \beta_{a n}^{(c)}\right), \\
& c=1, \ldots, N \\
& \sum_{\beta \in \mathcal{S}} p_{\beta} \leq 1 .
\end{aligned}
$$

Let $W_{\text {max }}(\hat{Q}(t))=\max _{\beta \in \mathcal{S}} W(\beta, \hat{Q}(t))$. For $t \geq 0$ we have

$$
\begin{aligned}
& W_{\text {max }}(\hat{Q}(t)) \geq \sum_{\beta \in \mathcal{S}} p_{\beta} W(\beta, \hat{Q}(t)) \\
& =\sum_{\beta \in \mathcal{S}} p_{\beta} \sum_{a=1}^{N} \sum_{b=1}^{N} \sum_{c=1}^{N} \beta_{a b}^{(c)}\left[\hat{Q}_{a}^{(c)}(t)-\hat{Q}_{b}^{(c)}(t)\right] \\
& =\sum_{\beta \in \mathcal{S}} p_{\beta} \sum_{n=1}^{N} \sum_{c=1}^{N} \hat{Q}_{n}^{(c)}(t)\left(\sum_{b=1}^{N} \beta_{n b}^{(c)}-\sum_{a=1}^{N} \beta_{a n}^{(c)}\right) \\
& =\sum_{n=1}^{N} \sum_{c=1}^{N} \hat{Q}_{n}^{(c)}(t) \cdot \sum_{\beta \in \mathcal{S}} p_{\beta}\left(\sum_{b=1}^{N} \beta_{n b}^{(c)}-\sum_{a=1}^{N} \beta_{a n}^{(c)}\right) \\
& =\sum_{n=1}^{N} \sum_{c=1}^{N} \hat{Q}_{n}^{(c)}(t) \cdot \lambda_{n}^{(c)} .
\end{aligned}
$$

Let $t$ be a fixed value such that $\hat{Q}(\cdot)$ is differentiable at $t$. Let $\mathcal{S}^{\prime}$ be the set of schedules $\beta$ such that $W(\beta, \hat{Q}(t))=W_{\max }(\hat{Q}(t))$. Then we have $\frac{d}{d t} W(\beta, \hat{Q}(t))=\frac{d}{d t} W_{\max }(\hat{Q}(t))$ for $\beta \in \mathcal{S}^{\prime}$ (see proof of Lemma 3.2 of Reference [9]). By Equations (16) and (18), we have

$$
\sum_{\beta \in \mathcal{S}^{\prime}} \frac{d}{d t} \hat{T}_{\beta}(t)=1 .
$$

It follows that

$$
\begin{aligned}
\sum_{n=1}^{N} & \sum_{c=1}^{N} \hat{Q}_{n}^{(c)}(t)\left(\sum_{b=1}^{N} \frac{d}{d t} \hat{D}_{n b}^{(c)}(t)-\sum_{a=1}^{N} \frac{d}{d t} \hat{D}_{a n}^{(c)}(t)\right) \\
= & \sum_{n=1}^{N} \sum_{c=1}^{N} \hat{Q}_{n}^{(c)}(t)\left(\sum_{b=1}^{N} \sum_{\beta \in \mathcal{S}^{\prime}} \beta_{n b}^{(c)} \frac{d}{d t} \hat{T}_{\beta}(t)\right. \\
& \left.-\sum_{a=1}^{N} \sum_{\beta \in \mathcal{S}^{\prime}} \beta_{a n}^{(c)} \frac{d}{d t} \hat{T}_{\beta}(t)\right) \\
= & \sum_{\beta \in \mathcal{S}^{\prime}} \frac{d}{d t} \hat{T}_{\beta}(t) \sum_{n=1}^{N} \sum_{c=1}^{N} \hat{Q}_{n}^{(c)}(t)\left(\sum_{b=1}^{N} \beta_{n b}^{(c)}-\sum_{a=1}^{N} \beta_{a n}^{(c)}\right)
\end{aligned}
$$




$$
\begin{aligned}
& =\sum_{\beta \in \mathcal{S}^{\prime}} \frac{d}{d t} \hat{T}_{\beta}(t) \sum_{a=1}^{N} \sum_{b=1}^{N} \sum_{c=1}^{N} \beta_{a b}^{(c)}\left[\hat{Q}_{a}^{(c)}(t)-\hat{Q}_{b}^{(c)}(t)\right] \\
& =\sum_{\beta \in \mathcal{S}^{\prime}} \frac{d}{d t} \hat{T}_{\beta}(t) \cdot W(\beta, \hat{Q}(t)) \\
& =W_{\text {max }}(\beta, \hat{Q}(t)) \sum_{\beta \in \mathcal{S}^{\prime}} \frac{d}{d t} \hat{T}_{\beta}(t) \\
& =W_{\text {max }}(\beta, \hat{Q}(t)) .
\end{aligned}
$$

Thus,

$$
\begin{aligned}
\frac{d}{d t} f(t)= & 2 \sum_{n=1}^{N} \sum_{c=1}^{N} \hat{Q}_{n}^{(c)}(t) \cdot \frac{d}{d t} \hat{Q}_{n}^{(c)}(t) \\
= & 2 \sum_{n=1}^{N} \sum_{c=1}^{N} \hat{Q}_{n}^{(c)}(t)\left(\lambda_{n}^{(c)}+\sum_{a=1}^{N} \frac{d}{d t} \hat{D}_{a n}^{(c)}(t)-\sum_{b=1}^{N} \frac{d}{d t} \hat{D}_{n b}^{(c)}(t)\right) \\
= & 2 \sum_{n=1}^{N} \sum_{c=1}^{N} \hat{Q}_{n}^{(c)}(t) \lambda_{n}^{(c)} \\
& -2 \sum_{n=1}^{N} \sum_{c=1}^{N} \hat{Q}_{n}^{(c)}(t)\left(\sum_{b=1}^{N} \frac{d}{d t} \hat{D}_{n b}^{(c)}(t)-\sum_{a=1}^{N} \frac{d}{d t} \hat{D}_{a n}^{(c)}(t)\right) \\
= & 2\left(\sum_{n=1}^{N} \sum_{c=1}^{N} \hat{Q}_{n}^{(c)}(t) \lambda_{n}^{(c)}-W_{\max }(\hat{Q}(t))\right) \\
\leq & 0 .
\end{aligned}
$$

In other words, we have $\frac{d}{d t} f(t) \leq 0$ for almost every $t$ such that $f$ is differentiable at $t$. Since $f(0)=0$, it follows that $f(t)=0$ for all $t \geq 0$ (see, for example, the proof of Lemma 1 of Reference [7]), proving the theorem.

\section{PROOF OF THEOREM 1}

Proof. It is sufficient to prove that each of the fluid limit paths satisfies Equation (18) if the system works under the L-BP policy. The proof is similar to the proof of Lemma 4 in Reference [8]. For completeness, we produce a full proof here.

Suppose $(\hat{Q}, \hat{D}, \hat{T})$ is a fluid limit path. Fix a sample path $\omega \in \Omega$ such that Equations (11) and (12) hold. There exists a sequence $\left\{r_{k}\right\}$ with $r_{k} \rightarrow \infty$ as $k \rightarrow \infty$, such that

$$
\begin{aligned}
&\left(\hat{Q}^{r_{k}}(\cdot, \omega), \hat{D}^{r_{k}}(\cdot, \omega), \hat{T}^{r_{k}}(\cdot, \omega)\right) \\
& \rightarrow(\hat{Q}, \hat{D}, \hat{T}) \quad \text { u.o.c } \quad \text { as } k \rightarrow \infty .
\end{aligned}
$$

Fix a time $t \geq 0$. Define $\mathcal{A}(\hat{Q}(t))=\left\{(a, c) \mid \hat{Q}_{a}^{(c)}(t)>0\right\}$. Let $\tilde{\alpha}=\operatorname{argmax}_{\alpha \in \mathcal{S}} W(\alpha, \hat{Q}(t))$. Define $\tilde{\beta}$ via

$$
\tilde{\beta}_{a b}^{(c)}= \begin{cases}\tilde{\alpha}_{a b}^{(c)} & \text { if }(a, c) \in \mathcal{A}(\hat{Q}(t)) \\ 0 & \text { otherwise }\end{cases}
$$


We have $\tilde{\beta} \in \mathcal{S}$, since $\tilde{\beta} \leq \tilde{\alpha}$. We now prove that $W(\tilde{\beta}, \hat{Q}(t))=\max _{\alpha \in \mathcal{S}} W(\alpha, \hat{Q}(t))$. Note that

$$
\begin{aligned}
W(\tilde{\alpha}, \hat{Q}(t))= & \sum_{a=1}^{N} \sum_{b=1}^{N} \sum_{c=1}^{N} \tilde{\alpha}_{a b}^{(c)}\left[\hat{Q}_{a}^{(c)}(t)-\hat{Q}_{b}^{(c)}(t)\right] \\
= & \sum_{a=1}^{N} \sum_{b=1}^{N} \sum_{c=1}^{N} \tilde{\alpha}_{a b}^{(c)}\left[\hat{Q}_{a}^{(c)}(t)-\hat{Q}_{b}^{(c)}(t)\right] \cdot 1_{\hat{Q}_{a}^{(c)}(t)=0} \\
& +\sum_{a=1}^{N} \sum_{b=1}^{N} \sum_{c=1}^{N} \tilde{\alpha}_{a b}^{(c)}\left[\hat{Q}_{a}^{(c)}(t)-\hat{Q}_{b}^{(c)}(t)\right] \cdot 1_{\hat{Q}_{a}^{(c)}(t)>0} \\
= & \sum_{a=1}^{N} \sum_{b=1}^{N} \sum_{c=1}^{N} \tilde{\alpha}_{a b}^{(c)}\left[0-\hat{Q}_{b}^{(c)}(t)\right] \cdot 1_{\hat{Q}_{a}^{(c)}(t)=0} \\
& +\sum_{a=1}^{N} \sum_{b=1}^{N} \sum_{c=1}^{N} \tilde{\alpha}_{a b}^{(c)}\left[\hat{Q}_{a}^{(c)}(t)-\hat{Q}_{b}^{(c)}(t)\right] \cdot 1_{\hat{Q}_{a}^{(c)}(t)>0} \\
\leq & \sum_{a=1}^{N} \sum_{b=1}^{N} \sum_{c=1}^{N} \tilde{\alpha}_{a b}^{(c)}\left[\hat{Q}_{a}^{(c)}(t)-\hat{Q}_{b}^{(c)}(t)\right] \cdot 1_{\hat{Q}_{a}^{(c)}(t)>0} \\
= & \sum_{a=1}^{N} \sum_{b=1}^{N} \sum_{c=1}^{N} \tilde{\beta}_{a b}^{(c)}\left[\hat{Q}_{a}^{(c)}(t)-\hat{Q}_{b}^{(c)}(t)\right] \cdot 1_{\hat{Q}_{a}^{(c)}(t)>0} \\
= & \sum_{a=1}^{N} \sum_{b=1}^{N} \sum_{c=1}^{N} \tilde{\beta}_{a b}^{(c)}\left[\hat{Q}_{a}^{(c)}(t)-\hat{Q}_{b}^{(c)}(t)\right] .
\end{aligned}
$$

Then we must have

$$
W(\tilde{\beta}, \hat{Q}(t))=W(\tilde{\alpha}, \hat{Q}(t))=\max _{\alpha \in \mathcal{S}} W(\alpha, \hat{Q}(t)) .
$$

Fix a schedule $\beta \in \mathcal{S}$ with $W(\beta, \hat{Q}(t))<W(\tilde{\beta}, \hat{Q}(t))$. There exists a constant $\epsilon>0$ such that

$$
W(\tilde{\beta}, \hat{Q}(t))-W(\beta, \hat{Q}(t))\rangle \geq \epsilon, \hat{Q}_{a}^{(c)}(t)>\epsilon \text { for }(a, c) \in \mathcal{A}(\hat{Q}(t)) .
$$

By the continuity of $\hat{Q}(\cdot)$, there exists $\tau>0$ such that for each $s \in[t-\tau, t+\tau]$

$$
\begin{aligned}
& W(\tilde{\beta}, \hat{Q}(s))-W(\beta, \hat{Q}(s)) \geq \frac{\epsilon}{2} \\
& \hat{Q}_{a}^{(c)}(s)>\frac{\epsilon}{2} \quad \text { for }(a, c) \in \mathcal{A}(\hat{Q}(s)) .
\end{aligned}
$$

Let $R$ be the maximal link speed all over the network as defined in Section 5.1. By Equation (23), there exists $K>0$ such that, for any $k>K$ we have $\frac{\epsilon}{4} r_{k}>\max \left(L_{\max }, N R\right)$ and for each $s \in[t-$ $\tau, t+\tau]$

$$
\begin{gathered}
\mid\left(W\left(\tilde{\beta}, \hat{Q}^{r_{k}}(s)\right)-W\left(\beta, \hat{Q}^{r_{k}}(s)\right)\right) \\
-(W(\tilde{\beta}, \hat{Q}(s))-W(\beta, \hat{Q}(s))) \mid \leq \frac{\epsilon}{4} \\
\left|\hat{Q}_{a}^{(c), r_{k}}(s)-\hat{Q}_{a}^{(c)}(s)\right| \leq \frac{\epsilon}{4}, \text { for }(a, c) \in \mathcal{A}(\hat{Q}(t)) .
\end{gathered}
$$


Thus for $k>K$ and each $s \in[t-\tau, t+\tau]$, we have

$$
\begin{aligned}
& W\left(\tilde{\beta}, \hat{Q}^{r_{k}}(s)\right)-W\left(\beta, \hat{Q}^{r_{k}}(s)\right) \geq \frac{\epsilon}{4} \\
& \hat{Q}_{a}^{(c), r_{k}}(s) \geq \frac{\epsilon}{4} \quad \text { for }(a, c) \in \mathcal{A}(\hat{Q}(t)) .
\end{aligned}
$$

Therefore, for each time $s \in\left[(t-\tau) r_{k},(t+\tau) r_{k}\right]$, we have

$$
\begin{gathered}
W(\tilde{\beta}, Q(s, \omega))>W(\beta, Q(s, \omega)), \\
Q_{a}^{(c)}(s, \omega) \geq \frac{\epsilon}{4} r_{k}>\max \left(L_{\text {max }}, N R\right) \quad \text { for }(a, c) \in \mathcal{A}(\hat{Q}(t)) .
\end{gathered}
$$

Condition (25) implies that schedule $\tilde{\beta}$ only serves queue that has queue backlog larger than $\max \left(L_{\text {max }}, N R\right)$ throughout time interval $\left[(t-\tau) r_{k},(t+\tau) r_{k}\right]$ and the queues it serves all have sufficient backlog to send. By Equation (6), it must be a valid schedule under the L-BP policy throughout time interval $\left[(t-\tau) r_{k},(t+\tau) r_{k}\right]$. By Equation (24), the weight of schedule $\beta$ is always less than that of $\tilde{\beta}$ and thus should never be employed throughout time interval $\left[(t-\tau) r_{k},(t+\tau) r_{k}\right]$. Therefore, for any $u_{1} \leq u_{2}, u_{1}, u_{2} \in\left[(t-\tau) r_{k},(t+\tau) r_{k}\right]$ we have

$$
T_{\beta}\left(u_{2}, \omega\right)-T_{\beta}\left(u_{1}, \omega\right)=0 .
$$

Thus, for any $u_{1}, u_{2} \in[(t-\tau),(t+\tau)]$ with $u_{1} \leq u_{2}$, we have

$$
T_{\beta}\left(u_{2} r_{k}, \omega\right)-T_{\beta}\left(u_{1} r_{k}, \omega\right)=0,
$$

i.e.,

$$
\hat{T}_{\beta}^{r_{k}}\left(u_{2}, \omega\right)-\hat{T}_{\beta}^{r_{k}}\left(u_{1}, \omega\right)=0 .
$$

Taking the limit as $k \rightarrow \infty$, we have

$$
\hat{T}_{\beta}\left(u_{2}\right)-\hat{T}_{\beta}\left(u_{1}\right)=0
$$

for any $u_{1}, u_{2} \in[(t-\tau),(t+\tau)]$ with $u_{1} \leq u_{2}$, from which Equation (18) follows, proving the lemma.

\section{PROOF OF THEOREM 2}

Proof. It is sufficient to prove that each of the fluid limit paths satisfies Equation (18) if the system works under the A-BP policy. The proof is quite similar to that of Theorem 1 .

Similarly, suppose $(\hat{Q}, \hat{D}, \hat{T})$ is a fluid limit path. Fix a sample path $\omega \in \Omega$ such that Equations (11) and (12) hold. There exists a sequence $\left\{r_{k}\right\}$ with $r_{k} \rightarrow \infty$ as $k \rightarrow \infty$, such that

$$
\begin{aligned}
&\left(\hat{Q}^{r_{k}}(\cdot, \omega), \hat{D}^{r_{k}}(\cdot, \omega), \hat{T}^{r_{k}}(\cdot, \omega)\right) \\
& \rightarrow(\hat{Q}, \hat{D}, \hat{T}) \quad \text { u.o.c } \quad \text { as } k \rightarrow \infty .
\end{aligned}
$$

Fix a time $t \geq 0$. Define $\mathcal{A}(\hat{Q}(t))=\left\{(a, c) \mid \hat{Q}_{a}^{(c)}(t)>0\right\}$. Let $\tilde{\alpha}=\operatorname{argmax}_{\alpha \in \mathcal{S}} W(\alpha, \hat{Q}(t))$. Define $\tilde{\beta}$ via

$$
\tilde{\beta}_{a b}^{(c)}=\left\{\begin{array}{ll}
\tilde{\alpha}_{a b}^{(c)} & \text { if }(a, c) \in \mathcal{A}(\hat{Q}(t)) \\
0 & \text { otherwise }
\end{array} .\right.
$$

We have $\tilde{\beta} \in \mathcal{S}$ and $W(\tilde{\beta}, \hat{Q}(t))=\max _{\alpha \in \mathcal{S}} W(\alpha, \hat{Q}(t))$.

Fix a schedule $\beta \in \mathcal{S}$ with $W(\beta, \hat{Q}(t))<W(\tilde{\beta}, \hat{Q}(t))$. There exists a constant $\epsilon>0$ such that

$$
W(\tilde{\beta}, \hat{Q}(t))-W(\beta, \hat{Q}(t))\rangle \geq \epsilon
$$


and $\hat{Q}_{a}^{(c)}(t)>\epsilon$ for $(a, c) \in \mathcal{A}(\hat{Q}(t))$. By the continuity of $\hat{Q}(\cdot)$, there exists $\tau>0$ such that for each $s \in[t-\tau, t+\tau]$

$$
\begin{aligned}
& W(\tilde{\beta}, \hat{Q}(s))-W(\beta, \hat{Q}(s)) \geq \frac{\epsilon}{2} \\
& \hat{Q}_{a}^{(c)}(s)>\frac{\epsilon}{2} \quad \text { for }(a, c) \in \mathcal{A}(\hat{Q}(s)) .
\end{aligned}
$$

Let $R$ be the maximal link speed all over the network as defined in Section 5.1. By Equation (26), there exists $K>0$ such that, for any $k>K$, we have $\frac{\epsilon}{4} r_{k}>\max \left(R A_{\max }, R N\right), \frac{\tau}{2} r_{k}>A_{\max }$ and for each $s \in[t-\tau, t+\tau]$

$$
\begin{gathered}
\mid\left(W\left(\tilde{\beta}, \hat{Q}^{r_{k}}(s)\right)-W\left(\beta, \hat{Q}^{r_{k}}(s)\right)\right) \\
-(W(\tilde{\beta}, \hat{Q}(s))-W(\beta, \hat{Q}(s))) \mid \leq \frac{\epsilon}{4} \\
\left|\hat{Q}_{a}^{(c), r_{k}}(s)-\hat{Q}_{a}^{(c)}(s)\right| \leq \frac{\epsilon}{4} \quad \text { for }(a, c) \in \mathcal{A}(\hat{Q}(t)) .
\end{gathered}
$$

Thus for $k>K$ and each $s \in[t-\tau, t+\tau]$, we have

$$
\begin{aligned}
& W\left(\tilde{\beta}, \hat{Q}^{r_{k}}(s)\right)-W\left(\beta, \hat{Q}^{r_{k}}(s)\right) \geq \frac{\epsilon}{4} \\
& \hat{Q}_{a}^{(c), r_{k}}(s) \geq \frac{\epsilon}{4} \quad \text { for }(a, c) \in \mathcal{A}(\hat{Q}(t)) .
\end{aligned}
$$

Therefore, for each time $s \in\left[(t-\tau) r_{k},(t+\tau) r_{k}\right]$, we have

$$
\begin{gathered}
W(\tilde{\beta}, Q(s))>W(\beta, Q(s)), \\
Q_{a}^{(c)}(s) \geq \frac{\epsilon}{4} r_{k}>\max \left(R A_{\text {max }}, R N\right), \text { for }(a, c) \in \mathcal{A}(\hat{Q}(t)) .
\end{gathered}
$$

Condition (28) implies that for $(a, c) \in \mathcal{A}(\hat{Q}(t))$, the length of queue $Q_{a}^{(c)}$ is always larger than $R A_{\max }$. Thus the delay of the head-of-line packet in $Q_{a}^{(c)}$ is always larger than $A_{\max }$ throughout time interval $\left[(t-\tau) r_{k}+A_{\max },(t+\tau) r_{k}\right]$. Since $\frac{\tau}{2} r_{k}>A_{\max }$, we have $\left[\left(t-\frac{\tau}{2}\right) r_{k},\left(t+\frac{\tau}{2}\right) r_{k}\right] \subset$ $\left[(t-\tau) r_{k}+A_{\max },(t+\tau) r_{k}\right]$. In other words, schedule $\tilde{\beta}$ only serves queue with delay of head-ofline packets larger than $A_{\max }$ throughout time interval $\left[\left(t-\frac{\tau}{2}\right) r_{k},\left(t+\frac{\tau}{2}\right) r_{k}\right]$ and the queues it serves all have sufficient backlog to send. By Equation (7), it must be a valid schedule under the A-BP policy throughout time interval $\left[\left(t-\frac{\tau}{2}\right) r_{k},\left(t+\frac{\tau}{2}\right) r_{k}\right]$. By Equation (27), the weight of schedule $\beta$ is always less than that of $\tilde{\beta}$ and thus should never be employed throughout time interval $\left[\left(t-\frac{\tau}{2}\right) r_{k},\left(t+\frac{\tau}{2}\right) r_{k}\right]$. Therefore, for any $u_{1} \leq u_{2}, u_{1}, u_{2} \in\left[\left(t-\frac{\tau}{2}\right) r_{k},\left(t+\frac{\tau}{2}\right) r_{k}\right]$, we have

$$
T_{\beta}\left(u_{2}, \omega\right)-T_{\beta}\left(u_{1}, \omega\right)=0 .
$$

Therefore, for any $u_{1}, u_{2} \in\left[\left(t-\frac{\tau}{2}\right),\left(t+\frac{\tau}{2}\right)\right]$ with $u_{1} \leq u_{2}$, we have

$$
T_{\beta}\left(u_{2} r_{k}, \omega\right)-T_{\beta}\left(u_{1} r_{k}, \omega\right)=0,
$$

i.e.,

$$
\hat{T}_{\beta}^{r_{k}}\left(u_{2}, \omega\right)-\hat{T}_{\beta}^{r_{k}}\left(u_{1}, \omega\right)=0 .
$$

Taking the limit as $k \rightarrow \infty$, we have

$$
\hat{T}_{\beta}\left(u_{2}\right)-\hat{T}_{\beta}\left(u_{1}\right)=0
$$

for any $u_{1}, u_{2} \in\left[\left(t-\frac{\tau}{2}\right),\left(t+\frac{\tau}{2}\right)\right]$ with $u_{1} \leq u_{2}$, from which Equation (18) follows, proving the theorem. 


\section{E PROOF OF THEOREM 3}

Proof. We first prove the throughput optimality of O-BP. It is sufficient to prove that each of the fluid limit paths satisfies Equation (18) if the system works under the O-BP policy. The proof is quite similar to that of Theorem 1.

Similarly, suppose $(\hat{Q}, \hat{D}, \hat{T})$ is a fluid limit path. Fix a sample path $\omega \in \Omega$ such that Equations (11) and (12) hold. There exists a sequence $\left\{r_{k}\right\}$ with $r_{k} \rightarrow \infty$ as $k \rightarrow \infty$, such that

$$
\begin{aligned}
\left(\hat{Q}^{r_{k}}(\cdot, \omega), \hat{D}^{r_{k}}(\cdot, \omega), \hat{T}^{r_{k}}(\cdot, \omega)\right) & \\
& \rightarrow(\hat{Q}, \hat{D}, \hat{T}) \quad \text { u.o.c as } k \rightarrow \infty .
\end{aligned}
$$

Fix a time $t \geq 0$. Define $\mathcal{A}(\hat{Q}(t))=\left\{(a, c) \mid \hat{Q}_{a}^{(c)}(t)>0\right\}$. Let $\tilde{\alpha}=\operatorname{argmax}_{\alpha \in \mathcal{S}} W(\alpha, \hat{Q}(t))$. Define $\tilde{\beta}$ via

$$
\tilde{\beta}_{a b}^{(c)}=\left\{\begin{array}{ll}
\tilde{\alpha}_{a b}^{(c)} & \text { if }(a, c) \in \mathcal{A}(\hat{Q}(t)) \\
0 & \text { otherwise }
\end{array} .\right.
$$

We have $\tilde{\beta} \in \mathcal{S}$ and $W(\tilde{\beta}, \hat{Q}(t))=\max _{\alpha \in \mathcal{S}} W(\alpha, \hat{Q}(t))$.

Fix a schedule $\beta \in \mathcal{S}$ with $W(\beta, \hat{Q}(t))<W(\tilde{\beta}, \hat{Q}(t))$. Similarly to the proof of Theorem 1, we can prove that there exist constants $\tau>0$ and $K>0$ such that, for any $k>K$ and time $s \in[(t-$ $\left.\tau) r_{k},(t+\tau) r_{k}\right]$, we have

$$
\begin{gathered}
W(\tilde{\beta}, Q(s, \omega))>W(\beta, Q(s, \omega)), \\
Q_{a}^{(c)}(s, \omega)>\max \left(H_{\text {max }}, N R\right) \quad \text { for }(a, c) \in \mathcal{A}(\hat{Q}(t)) .
\end{gathered}
$$

Condition (31) implies that schedule $\tilde{\beta}$ only serves per-destination queues with queue backlogs larger than $\max \left(H_{\text {max }}, N R\right)$ throughout time interval $\left[(t-\tau) r_{k},(t+\tau) r_{k}\right]$ and the queues it serves all have sufficient backlog to send. By Equation (8), it must be a valid schedule under the O-BP policy throughout time interval $\left[(t-\tau) r_{k},(t+\tau) r_{k}\right]$. This is because when Equation (31) holds, it also implies that $Z_{a}^{(j)}>H_{\max }$ for the corresponding output queue, as selected by OSPF. Therefore, for any $u_{1} \leq u_{2}, u_{1}, u_{2} \in\left[(t-\tau) r_{k},(t+\tau) r_{k}\right]$, we have

$$
T_{\beta}\left(u_{2}, \omega\right)-T_{\beta}\left(u_{1}, \omega\right)=0 .
$$

Similarly to Theorem 1 , we can then prove that

$$
\hat{T}_{\beta}\left(u_{2}\right)-\hat{T}_{\beta}\left(u_{1}\right)=0
$$

for any $u_{1}, u_{2} \in[(t-\tau),(t+\tau)]$ with $u_{1} \leq u_{2}$, from which Equation (18) follows, proving the throughput optimality of O-BP.

The proof for the throughput optimality of E-BP is almost the same to that of O-BP except for

$$
Q_{a}^{(c)}(s, \omega)>\max \left(M_{a}^{(c)} H_{\max }, N R\right) \quad \text { for }(a, c) \in \mathcal{A}(\hat{Q}(t))
$$

and substituting Equation (8) with Equation (9) in the above analysis under Inequality (32), where $M_{a}^{(c)} \leq M_{a}$ is the subset of all $M_{a}$ neighbors of node $a$ that are on a shortest path for commodity $c$. The analysis holds, because when Equation (32) holds, it also implies that $Z_{a}^{(j)}>H_{\text {max }}$ for at least one of the $M_{a}^{(c)}$ output queues, even if the content of $Q_{a}^{(c)}$ is evenly distributed over all $M_{a}^{(c)}$ output queues in the worst case. 


\section{REFERENCES}

[1] M. Alresaini, M. Sathiamoorthy, B. Krishnamachari, and M. J. Neely. 2012. Backpressure with adaptive redundancy (BWAR). In Proceedings of the 2012 Proceedings IEEE (INFOCOM'12). 2300-2308. DOI : https://doi.org/10.1109/INFCOM. 2012.6195617

[2] M. Alresaini, K. L. Wright, B. Krishnamachari, and M. J. Neely. 2016. Backpressure delay enhancement for encounterbased mobile networks while sustaining throughput optimality. IEEE/ACM Trans. Netw. 24, 2 (Apr. 2016), 1196-1208. DOI : https://doi.org/10.1109/TNET.2015.2404331

[3] Eleftheria Athanasopoulou, Loc X. Bui, Tianxiong Ji, R. Srikant, and Alexander Stolyar. 2013. Back-pressurebased packet-by-packet adaptive routing in communication networks. IEEE/ACM Trans. Netw. 21, 1 (2013) 244-257.

[4] B. Awerbuch and T. Leighton. 1993. A simple local-control approximation algorithm for multicommodity flow. In Proceedings of 1993 IEEE 34th Annual Foundations of Computer Science. 459-468. DOI : https://doi.org/10.1109/SFCS. 1993.366841

[5] Loc Bui, R. Srikant, and Alexander Stolyar. 2009. Novel architectures and algorithms for delay reduction in backpressure scheduling and routing. In Proceedings of the INFOCOM 2009, IEEE. IEEE, 2936-2940.

[6] Y. Cui, E. M. Yeh, and R. Liu. 2016. Enhancing the delay performance of dynamic backpressure algorithms. IEEE/ACM Trans. Netw. 24, 2 (Apr. 2016), 954-967. DOI : https://doi.org/10.1109/TNET.2015.2404852

[7] J. G. Dai and Balaji Prabhakar. 2000. The throughput of data switches with and without speedup. In Proceedings of the 19th Annual Joint Conference of the IEEE Computer and Communications Societies (INFOCOM'00), Vol. 2. IEEE, 556-564.

[8] Jim G. Dai and Wuqin Lin. 2005. Maximum pressure policies in stochastic processing networks. Operat. Res. 53, 2 (2005), 197-218.

[9] Jim G. Dai and Gideon Weiss. 1996. Stability and instability of fluid models for reentrant lines. Math. Operat. Res. 21, 1 (1996), 115-134.

[10] A. Eryilmaz and R. Srikant. 2006. Joint congestion control, routing, and MAC for stability and fairness in wireless networks. IEEE 7. Select. Areas Commun. 24, 8 (2006), 1514-1524

[11] Leonidas Georgiadis, Michael J. Neely, and Leandros Tassiulas. 2006. Resource allocation and cross-layer control in wireless networks. Found. Trends Netw. 1, 1 (2006), 1-144. DOI : https://doi.org/10.1561/1300000001

[12] C. Hopps. [n.d.]. Analysis of an equal-cost multi-path algorithm. Retrieved from http://tools.ietf.org/html/rfc $2992 /$.

[13] Internet2. [n.d.]. Advanced networking for leading-edge research and education. Retrieved from http://www. internet2.edu/.

[14] Sundar Iyer, Ramana Rao Kompella, and Nick McKeown. 2008. Designing packet buffers for router linecards. IEEE/ACM Trans. Netw. 16, 3 (2008), 705-717.

[15] Bo Ji, Changhee Joo, and Ness B. Shroff. 2013. Delay-based back-pressure scheduling in multihop wireless networks. IEEE/ACM Trans. Netw. 21, 5 (Oct. 2013), 1539-1552. DOI : https://doi.org/10.1109/TNET.2012.2227790

[16] Bo Ji, Changhee Joo, and Ness B. Shroff. 2013. Throughput-optimal scheduling in multihop wireless networks without per-flow information. IEEE/ACM Trans. Netw. 21, 2 (Apr. 2013), 634-647. DOI : https://doi.org/10.1109/TNET.2012 2205017

[17] J. Moy. [n.d.]. OSPF version 2. Retrieved from http://tools.ietf.org/html/rfc2328/.

[18] M. J. Neely, E. Modiano, and C. Li. 2005. Fairness and optimal stochastic control for heterogeneous networks. In Proceedings of IEEE Conference on Information Communications (INFOCOM'05). IEEE Press.

[19] Michael J Neely, Eytan Modiano, and Charles E Rohrs. 2005. Dynamic power allocation and routing for time-varying wireless networks. IEEE 7. Select. Areas Commun. 23, 1 (2005), 89-103.

[20] Jung Ryu, Lei Ying, and Sanjay Shakkottai. 2010. Back-pressure routing for intermittently connected networks. In Proceedings of the 29th Conference on Information Communications (INFOCOM'10). IEEE Press, 306-310. http://dl.acm. org/citation.cfm?id=1833515.1833577

[21] H. Seferoglu and E. Modiano. 2016. Separation of routing and scheduling in backpressure-based wireless networks. IEEE/ACM Trans. Netw. 24, 3 (Jun. 2016), 1787-1800. DOI : https://doi.org/10.1109/TNET.2015.2436217

[22] G. Shrimali and N. McKeown. 2005. Building packet buffers using interleaved memories. In Proceedings of the Workshop on High Performance Switching and Routing (HPSR'05). IEEE Press.

[23] L. Tassiulas and A. Ephremides. 1992. Stability properties of constrained queueing systems and scheduling policies for maximum throughput in multihop radio networks. IEEE Trans. Automat. Contr. 37, 12 (Dec. 1992), 1936-1948. DOI : https://doi.org/10.1109/9.182479

[24] Hao Wang and Bill Lin. 2010. Block-based packet buffer with deterministic packet departures. In Proceedings of the 11th International Conference on High Performance Switching (HPSR'10). IEEE Press.

[25] Hao Wang, Haiquan Zhao, Bill Lin, and Jun Xu. 2012. Robust pipelined memory system with worst case performance guarantee for network processing. IEEE/ACM Trans. Comput. 61, 10 (2012), 1386-1400. 
[26] Lei Ying, Sanjay Shakkottai, Aneesh Reddy, and Shihuan Liu. 2011. On combining shortest-path and back-pressure routing over multihop wireless networks. IEEE/ACM Trans. Netw. 19, 3 (2011), 841-854.

[27] Lei Ying, R. Srikant, and Don Towsley. 2008. Cluster-based back-pressure routing algorithm. In Proceedings of the Annual Conference on Information Communications (INFOCOM 2008). 484-492.

[28] Y. Zhang. [n.d.]. Abilene traffic matrices. Retrieved from http://www.cs.utexas.edu/ yzhang/research/AbileneTM/.

Received October 2017; revised April 2018; accepted July 2018 